\title{
Superradiant instability of extremal black holes in STU supergravity
}

\author{
Zhan-Feng Mai®, ${ }^{1}$ Run-Qiu Yang, ${ }^{1}$ and H. Lü $\odot^{1,2, *}$ \\ ${ }^{1}$ Center for Joint Quantum Studies and Department of Physics School of Science, Tianjin University, \\ Tianjin 300350, People's Republic of China \\ ${ }^{2}$ Peng Huangwu Center for Fundamental Theory, Hefei, Anhui 230026, People's Republic of China
}

(Received 9 November 2021; accepted 10 January 2022; published 27 January 2022)

\begin{abstract}
We investigate the superradiant (in)stability of the extremal multicharge static black holes in the STU supergravity model, which reduce to the Reissner-Nordström (RN) black hole when all the charges are equal. We first show that the frequency of quasibound states is necessarily complex and obtain the corresponding superradiant condition. We then study the effective potential of the Schrödinger-like equation associated with the radial function of the charged scalar field. We find that trapping-well configurations can emerge with either a single peak or double peaks. We numerically obtain the corresponding unstable quasibound states, organized under the overtone number, as well as how the charged black holes deviate from the RN black hole. We find that the STU black holes are superradiantly unstable as long as not all the charges are equal, indicating that the superradiant stability of the extremal RN black hole is a fine-tuning result in the framework of the STU supergravity model.
\end{abstract}

DOI: 10.1103/PhysRevD.105.024070

\section{INTRODUCTION}

With the detection of gravitational waves from two merging black holes and photo images of black holes $[1,2]$, an increasing amount of evidence suggests that black holes, predicted by Einstein's theory of general relativity, exist in our Universe. An important method to probe the black holes is to investigate the linear perturbations of matter or gravitational fields in the black hole background. These perturbations are typically described by secondorder differential equations. The nature of the black hole horizon requires that the boundary conditions of these linearized fields must be ingoing. We must also require that the fields are all finite at the asymptotic infinity. This leads to three inequivalent classes of asymptotic boundary conditions:

(i) Scattering processes: There are both ingoing and outgoing modes.

(ii) Quasinormal modes: There are outgoing modes only.

(iii) Quasibound states (QBS): The fields vanish. Specifically for a massive scalar particle field of mass $m_{p}$, if its frequency is sufficiently high $\left(\omega>m_{p}\right)$ so that it is free and wavelike in the asymptotic infinity, one can either

\footnotetext{
*mrhonglu@gmail.com
}

Published by the American Physical Society under the terms of the Creative Commons Attribution 4.0 International license. Further distribution of this work must maintain attribution to the author(s) and the published article's title, journal citation, and DOI. Funded by SCOAP. study the scattering process by not imposing any boundary condition, or study the quasinormal modes which reveal the (in)stability of the system by imposing only the outgoing boundary condition at infinity. When $\omega<m_{p}$, the wave functions either exponentially diverge or converge and the quasibound states are the latter case.

In a scattering process, we typically expect that the amplitude of an ingoing (incident) mode is bigger than that of the corresponding outgoing (reflection) mode. However, the opposite superradiance effect can arise in rotating or charged black holes [3-7], indicating that the scattering process can actually extract charges and/or angular momenta, and hence the energies from black holes. This phenomenon occurs for low-frequency excitations with $\omega<\omega_{c}$, where $\omega_{c}$ is a certain critical value that depends on the mass, angular momentum, and electric charge of the black hole. (See a review on superradiance [8].)

A brutal force implementation of the third boundary condition was first introduced by Press and Teukolsky [3]. They considered putting a mirror outside the black hole horizon such that the fields would all vanish asymptotically. It turns out the bounded scalar perturbation of a Kerr black hole can be exponentially amplified by extracting energy from the black hole, leading to a bomb effect. This black hole bomb mechanism was later realized naturally using massive scalar fields since they fall off exponentially at the asymptotic infinity for low-frequency excitations, leading to the superradiant instability of the Kerr or Kerr-Newmann black hole [9-13]. Interestingly, for asymptotic anti-de Sitter (AdS) spaceimes, the AdS boundary can provide such a "mirror" and superradiant 
instability can indeed arise in both charged static and rotating black holes [14-19]. In this paper, we shall consider only four-dimensional black holes that are asymptotic to the Minkowski spacetime.

Typically a black hole has three conserved quantities: the mass $M$, angular momentum $J$, and electric charge $Q$. For scalar field perturbations, it can be easily established that there is no superradiant effect or instability of neutral static black holes such as the Schwarzschild black hole. The superradiant effects were known to arise in both static and rotating black holes. There is hitherto no example of superradiant instability when $J=0$. Recently, it was shown that the Reissner-Nordström (RN) black hole is superradiantly stable [20-22]. Particularly, in [20], Hod examined the two necessary conditions for the existence of superradiant instability: (a) There exists an $\omega_{c}>0$ such that we can impose the superradiant condition $\omega<\omega_{c}$. (b) There exists a potential well to trap the quasibound states. It was shown that the two conditions could not be simultaneously satisfied for the extremal RN black hole.

In this paper, we study the superradiant instability of the $\mathrm{RN}$ black hole from the perspective of more fundamental theories such as strings and supergravities, where the extremal RN black hole emerges as a special Bogomol'nyiPrasad-Sommerfield (BPS) state. Specifically we consider STU supergravity, which is a special class of EinsteinMaxwell-dilaton theories involving four Maxwell fields and three dilaton scalars [23]. The theory admits a general class of four-charge black hole solutions and they reduce to the RN black hole when all the charges are exactly equal. The BPS (no-force) condition implies that in the extremal limit, the RN black hole can be viewed as a bound state with threshold binding energy of the four more fundamental ingredients that have origins as strings and branes in $D=10$ or 11 dimensions, e.g., [24]. We consider an additional massive scalar field charged under all the four Maxwell fields and we find that superradiant condition $\omega<\omega_{c}$ can be satisfied for the generic black hole charge configuration. When all the four black hole charges are equal, it can be demonstrated that there is no potential well when $\omega<\omega_{c}$. However, we find that a potential well can always form as long as the four charges of the extremal black hole are not all exactly the same. Furthermore, our numerical analysis indicates that the extremal STU black holes can all be superradiantly unstable provided that the black hole charges are not the same, regardless how small the difference is. Our results are the first in literature where the superradiant instability is demonstrated in spherically symmetric and static black holes.

The paper is organized as follows. In Sec. II, we briefly introduce the STU supergravity model and the associated extremal charged black hole with four $U(1)$ charges. By introducing a charged scalar perturbation, we obtain the radial wave equation, giving the boundary condition for the quasibound states. In Sec. III, we illustrate three necessary conditions for superradiant instability. We then analyze the conserved flux near the horizon for the superradiant condition and point out the equivalence between superradiant instability and unstable quasibound states. We give a no-go theorem of superradiant instability for the extremal $\mathrm{RN}$ black hole that emerges from four equal charges. In Sec. IV, we numerically construct the quasibound states by the shooting method and analyze the superradiant instability spectrum. Our results indicate that the extremal STU black holes are unstable as long as not all the charges are equal. In Sec. V, the conclusion and some further discussions are given. In Appendix A, we analyze the asymptotic boundary conditions of the radial wave function in tortoise coordinates. In Appendix B, we prove that the frequencies of quasibound states and also quasinormal modes are necessarily complex. We put most of the plots and graphs of numerical results in Appendix C.

\section{MODEL SETUP}

The STU supergravity model in four dimensions is the low-energy effective theory of six-dimensional string reduced on 2-torus [23]. The minimal field content of the bosonic sector consists of the metric $g_{\mu \nu}$ and four Maxwell fields $\left(\tilde{A}_{1}, \tilde{A}_{2}, \tilde{A}_{3}, \tilde{A}_{4}\right)$, in addition to the three dilatonic scalars and three axions that form three $\operatorname{SL}(2, \mathbb{R})$ complex scalars. For our purpose of studying the properties of electrically charged and static black holes, the axions decouple and the relevant Lagrangian is (e.g., [24])

$$
\begin{aligned}
\mathcal{L} & =\sqrt{-g}\left(R-\frac{1}{2}(\partial \vec{\varphi})^{2}-\frac{1}{4} \sum_{i=1}^{4} e^{\vec{a}_{i} \cdot \vec{\varphi}} \tilde{F}_{i}^{2}\right), \\
\vec{a}_{1} & =(1,1,1), \quad \vec{a}_{2}=(1,-1,-1), \quad \vec{a}_{3}=(-1,1,-1), \\
\vec{a}_{4} & =(-1,-1,1), \quad \vec{\varphi}=\left(\varphi_{1}, \varphi_{2}, \varphi_{3}\right), \quad \tilde{F}_{i}=d \tilde{A}_{i} .
\end{aligned}
$$

The theory admits an exact solution of static charged black holes. In the extremal limit, the solution is

$$
\begin{aligned}
d s^{2} & =-\left(\tilde{H}_{1} \tilde{H}_{2} \tilde{H}_{3} \tilde{H}_{4}\right)^{-\frac{1}{2}} d t^{2}+\left(\tilde{H}_{1} \tilde{H}_{2} \tilde{H}_{3} \tilde{H}_{4}\right)^{\frac{1}{2}}\left(d r^{2}+r^{2} d \Omega_{2}^{2}\right), \\
e^{\frac{1}{2} \vec{a}_{i} \cdot \vec{\varphi}} & =\tilde{H}_{i}^{-1}\left(\tilde{H}_{1} \tilde{H}_{2} \tilde{H}_{3} \tilde{H}_{4}\right)^{\frac{1}{4}}, \quad \tilde{A}_{i}=\left(\tilde{H}_{i}^{-1}-1\right) d t \\
\tilde{H}_{i} & =1+\frac{4 \tilde{Q}_{i}}{r^{2}}
\end{aligned}
$$

where $\tilde{Q}_{i}$ are the electric charges. The mass and entropy of these extremal black hole are

$$
\begin{gathered}
M=\tilde{Q}_{1}+\tilde{Q}_{2}+\tilde{Q}_{3}+\tilde{Q}_{4}, \\
S=16 \pi \sqrt{\tilde{Q}_{1} \tilde{Q}_{2} \tilde{Q}_{3} \tilde{Q}_{4} .}
\end{gathered}
$$

Note that $\tilde{Q}_{i}$ 's here are non-negative. When the electric charges $\tilde{Q}_{i}$ are all equal, the scalars all decouple and the 
solution becomes the extremal RN black hole. Thus in string theory, the RN black hole is not a fundamental object, but a bound state with threshold binding energy of fundamental ingredients that have higher dimensional origins as strings and branes. The reason we focus on extremal black holes is that they represent the balance between attractive and repulsive forces, and therefore the superradiant instability is most likely to occur.

The STU model can be further truncated to involve only one scalar and two Maxwell fields. Two situations can arise. One is to set $\tilde{A}_{1}=\tilde{A}_{2}$ and $\tilde{A}_{3}=\tilde{A}_{4}$ and the other is to set $\tilde{A}_{2}=\tilde{A}_{3}=\tilde{A}_{4}$. In both cases, the relevant Lagrangian can be cast into [25]

$\mathcal{L}=\sqrt{-g}\left(R-\frac{1}{2}(\partial \phi)^{2}-\frac{1}{4} \mathrm{e}^{\alpha_{1} \phi} F_{1}^{2}-\frac{1}{4} \mathrm{e}^{\alpha_{2} \phi} F_{2}^{2}\right)$,

where $F_{1}=\mathrm{d} A_{1}$ and $F_{2}=\mathrm{d} A_{2}$. The dilaton coupling constants $\left(\alpha_{1}, \alpha_{2}\right)$ satisfy

$\alpha_{1} \alpha_{2}=-1, \quad N_{1} \alpha_{1}+N_{2} \alpha_{2}=0, \quad N_{1}+N_{2}=4$.

The $\left(N_{1}, N_{2}\right)=(2,2)$ and $(1,3)$ cases reduce to the two above special simplified STU models, respectively. In particular, the $(1,3)$ case can be obtained from circle reduction of five-dimensional minimum supergravity, and $A_{1}$ is the Kaluza-Klein vector. Charged black holes for generic $\left(N_{1}, N_{2}\right)$ were obtained in [25] and the extremal solutions are

$$
\begin{aligned}
\mathrm{d} s^{2} & =-\left(H_{1}^{N_{1}} H_{2}^{N_{2}}\right)^{-\frac{1}{2}} \mathrm{~d} t^{2}+\left(H_{1}^{N_{1}} H_{2}^{N_{2}}\right)^{\frac{1}{2}}\left(\mathrm{~d} r^{2}+r^{2} \mathrm{~d} \Omega_{2}^{2}\right), \\
A_{i} & =\sqrt{N_{i}}\left(H_{i}^{-1}-1\right) \mathrm{d} t, \quad \phi=\sum_{i=1}^{2} \frac{1}{2} \alpha_{i} N_{i} \log H_{i}, \\
H_{i} & =1+\frac{4 Q_{i}}{\sqrt{N_{i}} r} .
\end{aligned}
$$

Note that when $\frac{Q_{1}}{\sqrt{N_{1}}}=\frac{Q_{2}}{\sqrt{N_{2}}}$, the above reduces to the extremal RN black hole, as we have discussed earlier. The truncated two-charge system is much simpler than the full STU model, while keeping the essential feature that the $\mathrm{RN}$ black hole is a bound state.

In order to study the superradiant instability, we consider a larger theory with additional massive scalar $\Phi$, charged under all the four $U(1)$ fields. The linear perturbation of this scalar is governed by its charged Klein-Gordon equation

$$
\begin{aligned}
\left(g^{\mu \nu} D_{\mu} D_{\nu}-m_{p}^{2}\right) \Phi= & 0 \\
D_{\mu}:= & \nabla_{\mu}-i \tilde{q}_{1} \tilde{A}_{1 \mu}-i \tilde{q}_{2} \tilde{A}_{2 \mu} \\
& -i \tilde{q}_{3} \tilde{A}_{3 \mu}-i \tilde{q}_{4} \tilde{A}_{4 \mu},
\end{aligned}
$$

in the background (2.2), where $\left(m_{p}, \tilde{q}_{i}\right)$ denote the fundamental mass and charges of the scalar. While we have $m_{p}>0$, the fundamental charges $\tilde{q}_{i}$ 's can be positive or negative, associated with particles or antiparticles. By contrast, $\tilde{Q}_{i}$ 's are all positive. It should be mentioned that although the charged massive scalar $\Phi$ can arise from the Kaluza-Klein reduction, it is expected to have a nonminimal coupling with the massless scalars of STU supergravity, as it does with the Kaluza-Klein vector. Therefore, the toy model (2.7) that we consider in this paper is likely to break the supersymmetry.

Since the background is spherically symmetric, static, and electrically charged, the general solution can be expressed as linear superpositions of different frequency modes:

$$
\Phi=\mathrm{e}^{-i \omega t} R(r) \mathrm{Y}_{\ell m}(\theta, \varphi),
$$

where $Y_{\ell, m}$ 's are spherical harmonics. The KG equation now reduces to

$$
-r^{2} \frac{\mathrm{d}}{\mathrm{d} r}\left(r^{2} \frac{\mathrm{d} R}{\mathrm{~d} r}\right)+U R=0
$$

with

$$
\begin{aligned}
U(r)= & \ell(\ell+1) r^{2}+m_{p}^{2} r^{4} \sqrt{\tilde{H}_{1} \tilde{H}_{2} \tilde{H}_{3} \tilde{H}_{4}} \\
& -r^{4}\left(\omega+\sum_{i=1}^{4} \tilde{q}_{i}\left(\tilde{H}_{i}^{-1}-1\right)\right)^{2} \tilde{H}_{1} \tilde{H}_{2} \tilde{H}_{3} \tilde{H}_{4} .
\end{aligned}
$$

Note that only $\ell$, not $m$, of $Y_{\ell, m}$ enters the reduced wave equation. In the regions of both the near horizon $(r \rightarrow 0)$ and infinity $(r \rightarrow \infty)$, the radial wave function $R(r)$ can be solved asymptotically:

$\left.R(r)\right|_{r \rightarrow 0} \sim \mathrm{e}^{i \frac{i\left(\omega-\omega_{c}\right)}{r}},\left.\quad R(r)\right|_{r \rightarrow \infty} \sim \frac{\mathrm{e}^{-\sqrt{m_{p}^{2}-\omega^{2}} r}}{r}$,

where

$$
\omega_{c} \equiv \tilde{q}_{1}+\tilde{q}_{2}+\tilde{q}_{3}+\tilde{q}_{4}
$$

Note that we have chosen the ingoing boundary condition on the horizon so that the coefficient "\#" of $\omega$ is positive. The $e^{i / r}$ factor above near the horizon $r \rightarrow 0$ is the characteristic wavelike behavior near the horizon of an extremal black hole, which in our case, has the $\mathrm{AdS}_{2} \times S^{2}$ near-horizon geometry. (For nonextremal black holes we would have $e^{i \log \left(r-r_{+}\right)}$instead.) It is important to note that we do not take the decoupling limit here and hence we do not probe the whole $\mathrm{AdS}_{2} \times S^{2}$ geometry.

The quantity $\omega_{c}$ is certain critical value that determines whether the black hole energy can be extracted; we shall discuss this presently. Here $\omega_{c}$ depends only on the fundamental charges; it is the sum of them all. At the 
asymptotic infinity, when $m_{p}<\omega$, the solution is wavelike, describing scattering processes in general, or quasinormal modes if we impose further the outgoing boundary condition asymptotically.

In this paper, we focus on the quasibound states where

$$
\omega<m_{p},
$$

such that the wave function falls off exponentially at the asymptotic infinity. It has a consequence, as in the case of quasinormal modes, that the frequency must be discrete. Furthermore, as we show in Appendix B, the frequency must be complex, namely

$$
\omega_{\mathrm{QBS}}=\omega_{r}+\mathrm{i} \omega_{i},
$$

where $\omega_{r}$ and $\omega_{i}$ denote the real and imaginary parts of $\omega_{\mathrm{QBS}}$, respectively. The existence of such bound states with positive $\omega_{i}$ indicates that they will grow exponentially with time and therefore the system is unstable at the linear level. The focus of this paper is to analyze this superradiant instability for the charged black holes in the STU model.

\section{SUPERRADIANT CONDITION FOR COMPLEX FREQUENCIES}

There are important differences between quasibound states(or quasinormal modes) and those associated with the scattering processes. In the latter case, the frequency $\omega$ is real, while the former typically involves complex frequencies. It is thus important to obtain the corresponding superradiant conditions of the two different situations.

\section{A. Superradiant condition for scattering processes}

We first review the superradiant condition for the scattering processes. As a concrete example, we illustrate this by using the extremal black hole of the STU model. The superradiant scattering is typically discussed in the tortoise coordinates, which we give in Appendix A. For the appropriately scaled radial wave function (A1), we have, with $m_{p}<\omega$,

$$
\bar{R}= \begin{cases}\mathcal{T} \mathrm{e}^{-i\left(\omega-\omega_{c}\right) y} & y \rightarrow-\infty \\ \mathcal{I} \mathrm{e}^{-i \sqrt{\omega^{2}-m_{p}^{2}} y}+\mathcal{R} \mathrm{e}^{i \sqrt{\omega^{2}-m_{p}^{2}} y} & y \rightarrow \infty,\end{cases}
$$

where $\omega_{c}$ for the extremal STU black holes is given by (2.12), and $\mathcal{I}, \mathcal{R}$, and $\mathcal{T}$ denote the incident, reflection, and transmission coefficients, respectively. The conservation of the Wroskian determinant associated with the radial wave function

$\left.W\right|_{y \rightarrow-\infty}=\left.W\right|_{y \rightarrow \infty}, \quad$ where $W=\bar{R}^{*} \frac{\mathrm{d} \bar{R}}{\mathrm{~d} y}-\bar{R} \frac{\mathrm{d} \bar{R}^{*}}{\mathrm{~d} y}$,

implies that

$$
|\mathcal{R}|^{2}=|\mathcal{I}|^{2}-\frac{\omega-\omega_{c}}{\sqrt{\omega^{2}-m_{p}^{2}}}|\mathcal{T}|^{2} .
$$

Thus when $\omega<\omega_{c}$, the reflecting amplitude is larger than the incident amplitude, namely $|\mathcal{R}|^{2}>|\mathcal{I}|^{2}$. It is important to note that in this scattering process, the frequency $\omega$ is real. The situation changes for quasibound states where $\omega$ is complex and we shall discuss this in the next subsection.

\section{B. Superradiant condition for quasibound states}

As shown in Appendix B, the frequency of the quasibound states is necessarily complex. The superradiant condition of the previous subsection is thus nonapplicable. In order to derive the new condition, we followed the observation [26] that the energy momentum tensor associated with the charged scalar perturbation is not conserved, but there still exists an energy conserved current. Specially, the energy momentum tensor associated with the complex scalar perturbation gives

$T_{\mu \nu}^{\Phi}=D_{(\mu} \Phi D_{\nu)}^{\dagger} \Phi^{\dagger}-\frac{1}{2} g_{\mu \nu}\left(D^{\rho} \Phi D_{\rho}^{\dagger} \Phi^{\dagger}-m_{p}^{2} \Phi \Phi^{\dagger}\right)$.

However, it is straightforward to verify that

$$
\begin{aligned}
\nabla^{\mu} T_{\mu \nu}^{\Phi}= & \left(\sum_{k=1}^{4} q_{k} \tilde{F}_{k \nu \rho}\right) \\
& \times\left(\sum_{j=1}^{4} \Phi^{\dagger} \Phi \tilde{q}_{j} \tilde{A}_{j}^{\rho}+i\left(\Phi^{\dagger} \nabla^{\rho} \Phi-\Phi \nabla^{\rho} \Phi^{\dagger}\right)\right) \neq 0 .
\end{aligned}
$$

The reason why it is not conserved is that at the perturbation level, the source term contributed by four $U(1)$ fields $\left(\tilde{A}_{1}, \tilde{A}_{2}, \tilde{A}_{3}, \tilde{A}_{4}\right)$ has been neglected. Following the method given in [26], we find a new "energy momentum tensor"

$$
\begin{aligned}
\tilde{T}_{\mu \nu}= & \sum_{j=r, i} 2 \nabla_{\mu} \Phi_{j} \nabla_{\nu} \Phi_{j}-g_{\mu \nu}\left(\nabla^{\rho} \Phi_{j} \nabla_{\rho} \Phi_{j}\right. \\
& \left.+\left(\sum_{i}^{4} \tilde{q}_{i} \tilde{A}_{i}^{\rho}\right)^{2} \Phi_{j}^{2}\right)-m_{p}^{2} \Phi_{j}^{2},
\end{aligned}
$$

where $\Phi_{r}=\frac{1}{2}\left(\Phi+\Phi^{\dagger}\right)$ and $\Phi_{i}=\frac{1}{2 i}\left(\Phi-\Phi^{\dagger}\right)$. Although this new energy momentum tensor does not satisfy the conservation law either, namely $\nabla^{\mu} \tilde{T}_{\mu \nu} \neq 0$, there does exist a conserved energy current that satisfies the conservation law, namely

$$
J_{\mathrm{E}}^{\mu}=\tilde{T}_{\nu}^{\mu}\left(\frac{\partial}{\partial t}\right)^{\nu}, \quad \nabla_{\mu} J_{\mathrm{E}}^{\mu}=0 .
$$


In the case of the quasibound states with complex frequencies, we can read off the growth rate of total energy outside of the horizon from $J_{\mathrm{E}}^{\mu}$ that

$$
\frac{\partial E}{\partial t}=2 \omega_{i} E, \quad \text { where } E=\int_{V} \sqrt{-g} J_{\mathrm{E}}^{0} .
$$

The growth rate of the total energy outside the horizon depends on the sign of the imaginary part of the quasibound state. A state is stable when its $\omega_{i} \leq 0$, but unstable when $\omega_{i}>0$.

We now derive a necessary condition for the superradiant instability. The conservation law Eq. (3.7) implies that

$$
\frac{\partial}{\partial t} \int_{V} J_{\mathrm{E}}^{0}=\left.C_{l m} J_{\mathrm{E}}^{r}\right|_{r \rightarrow 0} ^{r \rightarrow \infty},
$$

where $C_{l m}=\int Y_{l m} Y_{l m}^{*} \sin \theta \mathrm{d} \theta \mathrm{d} \varphi=\frac{4 \pi}{2 l+1}$. Recall that the radial wave function decays exponentially and it gives a vanishing energy current at the spatial infinity. Equation (3.8) and Eq. (3.9) give

$$
2 E \omega_{i} \propto-\left(|\omega|^{2}-\omega_{c} \omega_{r}\right),
$$

where the proportionality coefficient is positive. We therefore conclude that a necessary condition is

$$
\frac{|\omega|^{2}}{\omega_{r}}<\omega_{c}
$$

This condition however is not restrictive enough to guide our numerical calculation since it involves also the unknown $\omega_{i}$. When $\omega_{i}=0$, the above condition reduces to the one of the scattering process discussed earlier. However, for complex $\omega$, we can do better. This is because the superradiant condition derived from the energy current does not give any detail constraints on the conserved charge. The linear perturbation has also a charged conserved current defined as the Klein-Gordon product:

$$
i I_{Q}^{\mu}=\Phi^{\dagger} D^{\mu} \Phi-\Phi\left(D^{\mu} \Phi\right)^{\dagger},
$$

which satisfies the charged conservation law, $\nabla_{\mu} I_{Q}^{\mu}=0$. Similar to the case of energy flux, the growth rate of total charge outside the horizon can be given as

$$
\frac{\partial Q}{\partial t}=2 \omega_{i} Q, \quad \text { where } Q=\int_{V} \sqrt{-g} I_{Q}^{0} .
$$

Note that this is a global charge, not the black hole electric charges $\tilde{Q}_{i}$ or the scalars' fundamental charges $q_{i}$ 's. The growth rate of the total charge depends on the sign of $\omega_{i}$, whose positive values imply instability.

To obtain the condition for the associated superradiant condition, we adopt the charge conservation law $\nabla_{\mu} I^{\mu}=0$ and the Gauss law, giving

$$
\frac{\partial Q}{\partial t}=\left.C_{l m} \int_{V} I_{Q}^{r}\right|_{r \rightarrow 0} ^{r \rightarrow \infty} .
$$

Due to the exponential decay of the charged scalar leading to vanishing charged current at the spatial infinity, we arrive at

$$
2 Q \omega_{i} \propto-\left(\omega_{r}-\omega_{c}\right)
$$

For unstable states, we must have positive $\omega_{i}$; we thus obtain a practical condition for superradiant instability:

$$
\omega_{r}<\omega_{c}
$$

Note that this condition automatically implies the earlier condition (3.11). Since the quasibound states also require that $\omega<m_{p}$, the above condition does not constrain the relation between the scalar's mass $m_{p}$ and its fundamental charge $q$ that determines $\omega_{c}$. On the other hand, for scattering processes or quasinormal modes, the condition $\omega>m_{p}$ implies that the scalar must satisfy $m_{p}<q$ for it to experience the superradiant effect.

\section{Trapping-well condition}

In order to discuss superradiant instability, it is necessary to construct first the quasibound states. To study the existence of such states, it is instructive to cast Eq. (2.8) in terms of the Schrödinger-like equation. To do so, we define $\tilde{R}=r R$ such that the function $\tilde{R}$ satisfies the Schrödinger-like equation

$$
-\frac{d^{2} \tilde{R}}{d r^{2}}+V_{\mathrm{eff}} \tilde{R}=\omega^{2} \tilde{R},
$$

where $r \in(0, \infty)$ and the potential for the general fourcharge background (2.2) is

$$
\begin{aligned}
V_{\mathrm{eff}}= & \omega^{2}+\frac{U}{r^{4}} \\
= & \omega^{2}+m_{p}^{2} \sqrt{\tilde{H}_{1} \tilde{H}_{2} \tilde{H}_{3} \tilde{H}_{4}}-\left(\omega-\sum_{i=1}^{4} \frac{4 \tilde{q}_{i} \tilde{Q}_{i}}{r \tilde{H}_{i}}\right)^{2} \\
& \times \tilde{H}_{1} \tilde{H}_{2} \tilde{H}_{3} \tilde{H}_{4}+\frac{\ell(\ell+1)}{r^{2}} .
\end{aligned}
$$

Although it is not quite the same as the Schrödinger equation since the eigenvalue $\omega^{2}$ appears in the potential $V_{\text {eff }}$ as well, we expect that a bound state arises only when its corresponding potential has a trapping well. To study the shape of $V_{\text {eff }}(r)$, we note that 


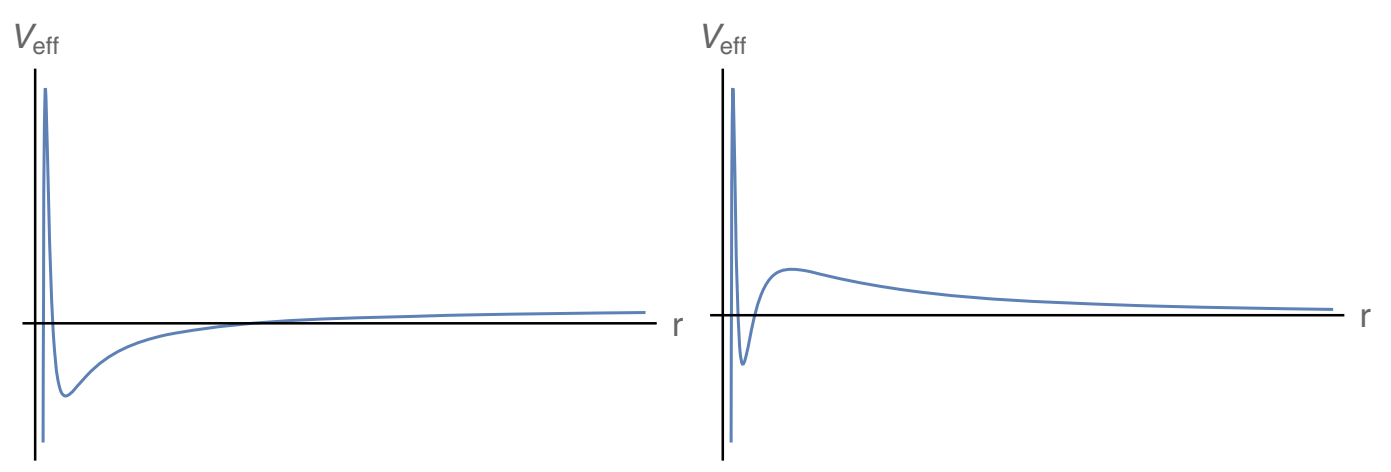

FIG. 1. Two types of trapping wells can arise in $V_{\text {eff }}$. The left has one peak; the well lies between the peak and the asymptotic infinity. The right has two peaks with the well sandwiched between.

$$
V_{\mathrm{eff}} \sim \begin{cases}\frac{\alpha}{r^{4}}, & r \rightarrow 0 ; \\ m_{p}^{2}+\frac{\beta}{r}, & r \rightarrow \infty,\end{cases}
$$

with

$$
\begin{aligned}
& \alpha=-256\left(\omega-\sum_{i=1}^{4} \tilde{q}_{i}\right)^{2} \prod_{i=1}^{4} \tilde{Q}_{i}, \\
& \beta=8 \sum_{i=1}^{4} \tilde{q}_{i} \tilde{Q}_{i}+2\left(m_{p}^{2}-2 \omega^{2}\right) \sum_{i=1}^{4} \tilde{Q}_{i} .
\end{aligned}
$$

Note that it is important that we take all $Q_{i}$ 's nonvanishing and hence $\alpha$ is nonzero. Thus we see that $\alpha<0$, but $\beta$ can be both positive or negative. The negative $\alpha$ is consistent with the fact that $r=0$ horizon is not the end of the space. It is a coordinate singularity, not an infinite potential barrier; it describes the other asymptotic region in the tortoise coordinates. For positive $\beta$, there can be at least one maximum for which $V_{\text {eff }}$ is positive. In general, there can be odd numbers of extrema and the existence of a trapping well requires at least three extrema. For negative $\beta$, there can be no extremum, or an even number of extrema. We find trapping wells with two extrema. We illustrate the shapes of $V_{\text {eff }}(r)$ of the two different types of trapping wells in Fig. 1.

The above discussion assumed that $\omega$ was real. However, as we show in Appendix B, the frequency for a quasibound state is necessarily complex. Thus we have in general

$$
V_{\text {eff }}=V_{\text {eff }}^{r}+i V_{\text {eff }}^{i}, \quad \omega^{2}=\left(\omega^{2}\right)_{r}+i\left(\omega^{2}\right)_{i} .
$$

The real and complex parts of the Schrödinger-like equation (3.17) are

$$
\begin{gathered}
-\frac{d^{2} \tilde{R}_{r}}{d r^{2}}+V_{\mathrm{eff}}^{r} \tilde{R}_{r}=\left(\omega^{2}\right)_{r} \tilde{R}_{r}+\left(V_{\mathrm{eff}}^{i}-\left(\omega^{2}\right)_{i}\right) \tilde{R}_{i}, \\
-\frac{d^{2} \tilde{R}_{i}}{d r^{2}}+V_{\mathrm{eff}}^{r} \tilde{R}_{i}=\left(\omega^{2}\right)_{r} \tilde{R}_{i}-\left(V_{\mathrm{eff}}^{i}-\left(\omega^{2}\right)_{i}\right) \tilde{R}_{r},
\end{gathered}
$$

where $\tilde{R}_{r}+i \tilde{R}_{i}=\tilde{R}$. It is clear that for real $\omega$, both $R_{r}$ and $R_{i}$ independently satisfy the same equation. They become coupled equations when $\omega_{i}$ is nonvanishing. Our numerical analysis indicates that $\omega_{i} / \omega_{r}<10^{-6}$ for all the bound states. Therefore, in the coupled equations, $R_{r}$ and $R_{i}$ provide perturbative sources for the same leading equation for $R_{i}$ and $R_{r}$, respectively. In this perturbative approach, the existence of trapping well can be determined by considering the real $\omega$ only.

\section{No superradiant instability of the $\mathbf{R N}$ black hole}

Having determined the necessary conditions for the superradiant instability, we can search for bound-state solutions of (3.17). This in general requires numerical calculations. However, in some simpler cases, we can determine from the effective potential that bound states are nonexisting. Such an example is provided by the RN black hole, which arises from the STU model by taking $\tilde{Q}_{1}=\tilde{Q}_{2}=\tilde{Q}_{3}=\tilde{Q}_{4}=Q / 2$. As was argued previously, we consider the case with real $\omega$. The bound-state boundary condition and superradiant condition are

$$
\omega<m_{p}, \quad \omega<\omega_{c} .
$$

To examine the shape of $V_{\text {eff }}$, we define two positive real numbers $(x, y)$ :

$$
m_{p}=\omega(1+x), \quad \omega_{c}=\omega(1+y) .
$$

We find that the effective potential becomes

$$
\begin{aligned}
V_{\mathrm{eff}}= & (x+1)^{2} \omega^{2}+\frac{4 Q \omega^{2}\left(x^{2}+2 x+y\right)}{r} \\
& +\frac{\ell(\ell+1)+4 Q^{2} \omega^{2}\left(x^{2}+2 x-(y-4) y\right)}{r^{2}} \\
& -\frac{16 Q^{3}(y-1) y \omega^{2}}{r^{3}}-\frac{16 Q^{4} y^{2} \omega^{2}}{r^{4}} .
\end{aligned}
$$

The bound-state condition and superradiant condition require that 


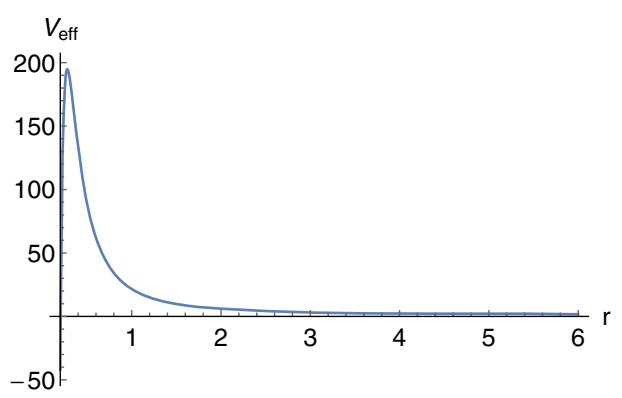

FIG. 2. As an illustration, we plot the effective potential of the scalar radial wave in the extremal RN black hole for $\ell=1$, $m_{p}=1, \omega=0.9, \tilde{q}_{i}=\frac{1}{4}$, and $Q=2$. This feature of having one extremal turns out to be generic when both the $\omega<m_{p}$ and $\omega<$ $\omega_{c}$ conditions are satisfied.

$$
x>0, \quad y>0 .
$$

We see that the leading $1 / r$ falloff at large $r$ is positive and the potential is negative as $r \rightarrow 0$. This implies that we must have at least three extrema at $r>0$ in order to have any bound states. We find

$$
\begin{aligned}
V_{\mathrm{eff}}^{\prime} & =\frac{-4 Q \omega^{2}\left(x^{2}+2 x+y\right)}{r^{5}} U, \\
U & =r^{3}+a_{2} r^{2}+a_{1} r+a_{0},
\end{aligned}
$$

where

$$
\begin{aligned}
& a_{2}=\frac{\ell(\ell+1)+4 Q^{2} \omega^{2}\left(x^{2}+2 x-(y-4) y\right)}{2 Q \omega^{2}\left(x^{2}+2 x+y\right)}, \\
& a_{1}=-\frac{12 Q^{2}(y-1) y}{x^{2}+2 x+y}, \quad a_{0}=-\frac{16 Q^{3} y^{2}}{x^{2}+2 x+y} .
\end{aligned}
$$

It is clear that $U$ has three roots $\left(r_{1}, r_{2}, r_{3}\right)$. If these three roots are all positive, then we must have

$$
a_{0}<0, \quad a_{1}>0, \quad a_{2}<0 .
$$

The first equality is automatically satisfied, but

$$
\begin{cases}a_{1}>0: & \rightarrow y<1 \\ a_{2}<0: & \rightarrow y>4\end{cases}
$$

Thus the conditions for having three real positive roots cannot be satisfied for this case.

As an example, the associated effective potential $V_{\text {eff }}$ for $\ell=1, m_{p}=1, \omega=0.9, \tilde{q}_{i}=\frac{1}{4}$, and $Q=2$ is plotted in Fig. 2. It is obvious that even though the superradiant condition and bound-state condition Eq. (3.23) are satisfied, the superradiant instability will not be triggered on since there is no trapping well for the effective potential. Of course, the true bound states require that the frequency $\omega$ be complex, in which case the discussion is far more complicated. However, our numerical analysis indicates, for all the bound states associated with the STU charged black holes, that $\omega_{i} / \omega_{r}<10^{-6}$ and therefore the above conclusion is valid.

The absence of a trapping well in the effective potential illustrates that the interaction or the force between the charged scalar and the RN black hole is too "simply" structured involving only the gravitational attraction and charge repulsion. In order to create a trapping well, additional attractive force is necessarily outside the horizon of the black hole. This is one of the reasons why we study STU black holes which involve not only the Maxwell fields but also the dilatonic scalar fields that can give nontrivial interactions and therefore create possibly the needed trapping wells.

\section{SUPERRADIANT INSTABILITY OF STU BLACK HOLES}

In the previous section, we show that in the RN black hole limit where all the charges are equal, there is no superradiant instability, regardless of the configuration of the fundamental charges $\tilde{q}_{i}$. In this section, we show that superradiance can develop instability when $\tilde{Q}_{i}$ are not all equal. For simplicity, we shall consider the simplified twocharge solution Eq. (2.6). In this case, the radial equation takes the same form of (2.9), but with

$$
\begin{aligned}
U(r)= & -r^{4} H_{1}^{N_{1}} H_{2}^{N_{2}}\left(\omega-\sum_{i=1}^{2} \frac{4 \sqrt{N_{i}} q_{i} Q_{i}}{r H_{i}}\right)^{2} \\
& +m_{p}^{4} r^{4} H_{1}^{N_{1} / 2} H_{2}^{N_{2} / 2}+\ell(\ell+1) r^{2} .
\end{aligned}
$$

In addition, the effective potential in the Schrödinger-like equation (3.17) is given by

$$
\begin{aligned}
V_{\text {eff }}= & \omega^{2}+m_{p}^{2} \sqrt{H_{1}^{N_{1}} H_{2}^{N_{2}}}-\left(\omega-\sum_{i=1}^{2} \frac{4 \sqrt{N_{i}} q_{i} Q_{i}}{r H_{i}}\right)^{2} \\
& \times H_{1}^{N_{1}} H_{2}^{N_{2}}+\frac{\ell(\ell+1)}{r^{2}},
\end{aligned}
$$

where we require that the frequency $\omega$ satisfy both the bound-state condition and superradiant condition:

$$
\omega<m_{p}, \quad \omega<\omega_{c}=\sqrt{N_{1}} q_{1}+\sqrt{N_{2}} q_{2} .
$$

In the RN black hole limit, $Q_{1} / \sqrt{N_{1}}=Q_{2} / \sqrt{N_{2}}$, it is straightforward to show that $V_{\text {eff }}$ has no trapping well and hence there is no superradiant instability.

\section{A. Effective potentials with trapping well}

The effective potential with trapping-well configuration does arise when the four charges in the STU model are not all equal. In this paper, we shall focus on the 
$\left(N_{1}, N_{2}\right)=(2,2)$ and $(1,3)$ examples that are the special cases in STU supergravity. As was mentioned earlier, there are two types of trapping wells, shown in Fig. 1, depending on whether the leading falloff parameter $\beta$ of the potential (3.19) is positive or negative. Here we present some concrete examples.

If we choose parameters $\left(\omega, m_{p}, q_{1}, q_{2}, \ell, Q_{1}, Q_{2}\right)=$ $(18 / 100,2 / 10,2 / 10,0,1,1,8)$, the potential has two extrema for both $\left(N_{1}, N_{2}\right)=(2,2)$ and $(1,3)$ cases. In the former case, the peak $V_{\mathrm{eff}}^{\max }=2.93$ at $r=1.34$ and the trapping-well minimum $V_{\text {eff }}^{\min }=-0.0326$ at $r=7.27$. In the latter case, we have $V_{\text {eff }}^{\max }=51.99$ at $r=0.39$ and $V_{\text {eff }}^{\min }=-6.14$ at $r=1.24$. The potentials all have the shape of the left panel in Fig. 1.

If we choose the corresponding parameters as (499/1000, $5 / 10,2 / 10,2 / 10,1,10,1 / 10)$, the potential has instead three extrema: one trapping-well minimum sandwiched between two peaks. For $\left(N_{1}, N_{2}\right)=(2,2)$ the peaks 685 and 0.26 are located at $r=0.07$ and 68.33 , with the minimum -192 at 0.21 . For $\left(N_{1}, N_{2}\right)=(1,3)$, the minimum -0.11 at 1.51 is sandwiched between the two peaks 3983 and 0.29 located at 0.019 and 25.22 respectively. These potentials have the shape of the right panel in Fig. 1. encouraging sign, it does not guarantee that quasibound states must exist, since the existence depends on both the width and depth of the trapping well. We therefore adopt a
While the existence of a trapping-well potential is an

numerical approach next to search for the quasibound states in the parameter space where trapping wells arise.

\section{B. Numerical setup: The shooting method}

Here we briefly discuss our numerical approach for calculating the quasibound states. While the Schrödingerlike equation (3.17) guides us to find the parameter regions where solutions may exist, we actually use Eq. (2.9) and its boundary condition Eq. (2.11) to construct explicit numerical solutions. We adopt the shooting method to perform numerical calculations. As shown in Appendix B, the frequency of these states must be complex, $\omega=\omega_{r}+i \omega_{i}$, and the sign of $\omega_{i}$ plays a defining role whether a state is stable or unstable. However, the imaginary part of the frequency is generally small, compared to its real part. (See also the case of Kerr black hole in [12].) In order to avoid the cutoff error, accuracy issues must be taken care of in order to obtain the trustworthy results.

First we take the following asymptotic ansatz of the radial function as the boundary conditions near the horizon and asymptotic infinity:

$$
\begin{aligned}
& \left.R(r \rightarrow 0) \sim \mathrm{e}^{i \frac{\chi_{1}\left(\omega-\omega_{c}\right)}{r}} r^{-2 i \chi_{2}} \sum_{i=0}^{n_{1}} r^{i} h_{i}\right|_{r=\epsilon_{c}}, \\
& \left.R(r \rightarrow \infty) \sim \mathrm{e}^{-k r} r^{\chi_{3}} \sum_{i=0}^{n_{2}} \frac{g_{i}}{r^{i}}\right|_{r=r_{c}},
\end{aligned}
$$

where

$$
\begin{aligned}
\chi_{1} & =16 N_{1}^{-N_{1} / 4} N_{2}^{-N_{2} / 4} Q_{1}^{N_{1} / 2} Q_{2}^{N_{2} / 2}, \\
\chi_{2} & =N_{1}^{-N_{1} / 4} N_{2}^{-N_{2} / 4} Q_{1}^{-1+N_{1} / 2} Q_{2}^{-1+N_{2} / 2}\left(2 N_{2} Q_{1} q_{2}+2 N_{1} q_{1} Q_{2}+\left(N_{2}^{3 / 2} Q_{1}+N_{1}^{3 / 2} Q_{2}\right)\left(\omega-\omega_{c}\right)\right), \\
\chi_{3} & =1-2 k\left(\sqrt{N_{1}} Q_{1}+\sqrt{N_{2}} Q_{2}\right)+\frac{m_{p}^{2}\left(\sqrt{N_{1}} Q_{1}+\sqrt{N_{2}} Q_{2}\right)-4\left(q_{1} Q_{1}+q_{2} Q_{2}\right) \omega}{k}, \\
k & =\sqrt{m_{p}^{2}-\omega^{2}}, \quad \omega_{c}=\sqrt{N_{1}} q_{1}+\sqrt{N_{2}} q_{2} .
\end{aligned}
$$

Since the equation is linear, we set $h_{0}=1=g_{0}$ without loss of generality, and we can obtain the coefficients $\left(h_{i}, g_{i}\right)$ analytically by solving the radial equation order by order. We present the explicit $\left(h_{1}, g_{1}\right)$ here:

$$
\begin{aligned}
h_{1}= & \frac{i}{32\left(\omega-\omega_{c}\right)}\left(16 m_{p}^{2}+\frac{16\left(\ell(\ell+1)+2 \chi_{2}\left(i+2 \chi_{2}\right)\right)}{\chi_{1}}-\frac{\left(N_{1} N_{2}\right)^{3 / 2} \chi_{1}}{Q_{1} Q_{2}}\left(\omega^{2}-2 \omega\left(q_{2}\left(N_{2}-1\right)\left(\frac{1}{\sqrt{N_{2}}}-\frac{Q_{1}}{N_{1}^{3 / 2} Q_{2}}\right)\right.\right.\right. \\
& \left.\left.+q_{1}\left(N_{1}-1\right)\left(\frac{1}{\sqrt{N_{1}}}-\frac{Q_{2}}{N_{2}^{3 / 2} Q_{1}}\right)\right)\right)+q_{1}^{2}\left(\frac{\sqrt{N_{1}} Q_{2}\left(2 N_{1}-3\right)}{N_{2}^{3 / 2} Q_{1}}-N_{1}+2\right)+q_{2}^{2}\left(\frac{\sqrt{N_{2}} Q_{1}\left(2 N_{2}-3\right)}{N_{1}^{3 / 2} Q_{2}}-N_{2}+2\right) \\
& \left.+2 q_{1} q_{2}\left(\frac{\left(N_{1}-1\right) Q_{2}}{N_{2} Q_{1}}+\frac{\left(N_{2}-1\right) Q_{1}}{N_{1} Q_{2}}-\frac{N_{1} N_{2}-3}{\sqrt{N_{1} N_{2}}}\right)\right), \\
g_{1}= & \frac{1}{2 k}\left(\ell(\ell+1)-\chi_{3}\left(1-2 k\left(\sqrt{N_{1}} Q_{1}+\sqrt{N_{2}} Q_{2}+\chi_{3}\right)\right)-\frac{8\left(\sqrt{N_{1}} Q_{2}-\sqrt{N_{2}} Q_{1}\right)\left(N_{1} q_{1} Q_{2}-N_{2} q_{1} Q_{1}\right) \omega}{\sqrt{N_{1} N_{2}}}\right. \\
& \left.-\left(4\left(Q_{1}^{2}+Q_{2}^{2}\right)\left(k^{2}-\omega^{2}\right)+\left(2\left(\sqrt{N_{1}} Q_{1}+\sqrt{N_{2}} Q_{2}\right) \omega-4\left(q_{1} Q_{1}+q_{2} Q_{2}\right)\right)^{2}\right)\right) .
\end{aligned}
$$


The expressions for higher-order coefficients become increasingly complicated and we shall not give them here. These power series expansions are necessary since the radial equation is singular on the $r=0$ horizon and we cannot either take the $r=\infty$ boundary condition literally in the numerical approach. We denote $\epsilon_{c}$ and $r_{c}$ as the numerical cutoffs for the horizon and asymptotic infinity, respectively. In general, the cutoff error can be numerically controlled under the maximum of $\mathcal{O}\left(\epsilon_{c}^{n_{1}+1}\right)$ and $\mathcal{O}\left(\frac{1}{r_{c}^{n_{2}+1}}\right)$ by the expansion power $\left(n_{1}, n_{2}\right)$. For the larger orders $\left(n_{1}, n_{2}\right)$ of the power expansions, we can use the smaller integration range $\left[\epsilon_{c}, r_{c}\right]$ for the desired accuracy.

Using the Runge-Kutta method, we integrate numerically from $\epsilon_{c}$ to some midpoint $r_{i}$, obtaining a numerical solution $R_{1}$; we also obtain the solution $R_{2}$ by integrating from $r_{c}$ to $r_{i}$. If $R_{1}$ and $R_{2}$ describe the same solution in different regions, then they should match at $r_{i}$. To match the solutions obtained from different regions, we require the Wronskian of $R_{1}, R_{2}$ at the middle point $r_{i}$ to vanish, namely,

$$
W\left(R_{1}, R_{2}\right)=\left.\frac{R_{1} R_{2}^{\prime}-R_{2} R_{1}^{\prime}}{\left|R_{1}\right|\left|R_{2}\right|}\right|_{r=r_{i}}=0 .
$$

[It is important to note that the matching condition is invariant under $R_{i} \rightarrow c_{i} R_{i}$ for any nonvanishing constant $\left(c_{1}, c_{2}\right)$; therefore, we could have chosen $h_{0}=1=g_{0}$ without loss of generality.] To implement this matching condition numerically, we consider an appropriate small dimensionless number $W_{c}$ such that we require

$$
\left.r W\left(R_{1}, R_{2}\right)\right|_{r=r_{i}}<W_{c} .
$$

Having established the shooting method for calculating the quasibound states, we can start by selecting a real $\omega$ such that the corresponding $V_{\text {eff }}$ has a trapping well. We use a numerical finding root program to obtain the correct complex $\omega_{\mathrm{QBM}}$ that satisfies the numerical Wronskian condition (4.8). We then incrementally scan the $\omega$ in an appropriate range and obtain the spectrum of the quasibound states. We can set $\omega_{r}$ positive without loss of generality, and we look for solutions with positive $\omega_{i}$ such that the wave function has an exponentially increasing factor $e^{\omega_{i} t}$, signaling superradiant instability.

Our numerical analysis indicates that the frequency ratio $\varpi \equiv \omega_{i} / \omega_{r}$ is smaller than $10^{-6}$, and therefore we need to increase our numerical accuracy so that the results are trustworthy. However, the higher accuracy requirement when $\varpi$ is about $10^{-13}$ makes the calculation timeconsuming. We thus set the cutoffs of the near horizon and the infinity to be $\epsilon_{h} \sim 10^{-2}$ and $r_{c} \sim 10^{2}$, respectively, and take the expansion order $\left(n_{1}, n_{2}\right)=(10,10)$. This leads to the cutoff errors under $10^{-21}$. Furthermore, we take the numerical Wronskian condition parameter
$W_{c} \sim 10^{-16}$. We believe that this ensures that $\varpi$ of the quasibound states can be numerically calculated within sufficient accuracy in the range $10^{-6} \sim 10^{-13}$. We carry out these calculation for both $\left(N_{1}, N_{2}\right)=(2,2)$ and $(1,3)$ cases and present the results next.

\section{Numerical Results}

In this subsection, we present our numerical results of quasibound states with superradiant instability. We set $\ell=1$ for all our numerical calculation. It is useful to define a dimensionless parameter $\epsilon$ that measures the deviation of the STU black hole from the RN black hole:

$$
\epsilon \equiv \frac{\sqrt{N_{1}} Q_{2}}{\sqrt{N_{2}} Q_{1}}-1 .
$$

The RN black hole corresponds to $\epsilon=0$, for which there is no superradiant instability. The mass of the extremal black hole is then given by

$$
M=\frac{Q_{1}}{\sqrt{N_{1}}}\left(4+N_{2} \epsilon\right) .
$$

It is clear that $\epsilon$ lies in the region $-1<\epsilon<\infty$. The purpose of this paper is not to be exhaustive and we focus on the cases with $\epsilon>0$. (When $N_{1}=N_{2}=2$, the negative region of $\epsilon$ is equivalent to that of $\epsilon>0$.)

\section{Effective potential with single peak}

In this subsection, we present the partial spectrum of the quasibound states with superradiant instability, arising from the trapping-well potential with a single peak. In other words, we choose parameters such that the potential $V_{\text {eff }}$ has the shape of the left panel in Fig. 1. As in the case of the Schrödinger equation where the energy is quantized for bound states, the complex frequency of our classical quasibound states are also quantized. The characteristics of the quantization can be described by the "overtone" number $n$, which counts the number of the peaks of the radial wave function $|R(r)|$. The $n=1,2, \ldots$, correspond to the ground state, first-excited and second-excited states, etc.

In Fig. 3 of Appendix C, we plot some explicit low-lying examples of $|R(r)|(n=1,2,3)$ for various parameters. In all the plots we have fixed $m_{p}=\frac{2}{10}, q_{1}=\frac{2}{10}, q_{2}=0$, and $Q_{1}=1$. (Recall that we set $\ell=1$ for all our numerical calculation.) Note that in these plots we have normalized radial wave equation by

$$
\int_{\epsilon_{h}}^{r_{c}} \sqrt{-\bar{g}}|R(r)|^{2}=1,
$$

where $\bar{g}$ denotes the determinant of the background metric. Although we present $|R|$ graphs for $n=1,2,3$, we actually 

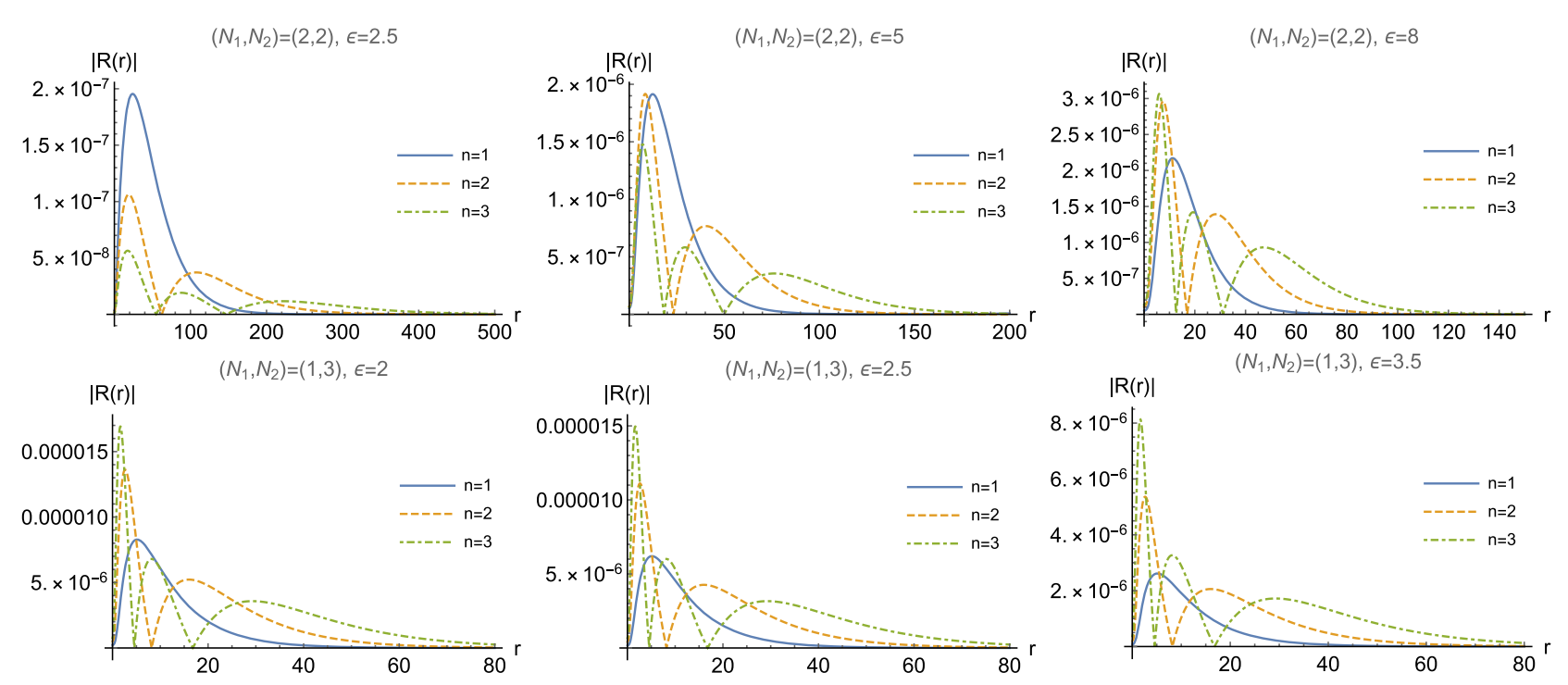

FIG. 3. Here we plot the normalized radial function $|R(r)|$ for low-lying quasibound states $(n=1,2,3)$ for black hole of various parameters. These states are characterized by that the radial function falls off exponentially as $r \rightarrow \infty$. Fixed parameters are $m_{p}=\frac{2}{10}$, $q_{1}=\frac{2}{10}, q_{2}=0$, and $Q_{1}=1$.

obtained the results for all the low-lying examples up to and including $n=10$. The complex frequencies of these quasibound states are summarized in Tables I and II.
In a quantum-mechanical system, $\omega_{r}$ would be the quantized energy of the system and the overtone number $n$ would describe the energy level. For our classical

TABLE I. The explicit complex frequencies of low-lying overtone states with $m_{p}=\frac{2}{10}, q_{1}=\frac{2}{10}, q_{2}=0$, and $Q_{1}=1$ in the $\left(N_{1}, N_{2}\right)=$ $(2,2)$ case.

\begin{tabular}{llcc}
\hline \hline$n$ & $\epsilon=2.5$ & $\epsilon=5$ & $\epsilon=8$ \\
\hline 1 & $0.19568+1.8307 \times 10^{-8} i$ & $0.18108+4.0846 \times 10^{-7} i$ & $0.16555+5.5423 \times 10^{-7} i$ \\
2 & $0.19773+1.2333 \times 10^{-9} i$ & $0.18741+5.9368 \times 10^{-7} i$ & $0.17332+1.8124 \times 10^{-6} i$ \\
3 & $0.19864+7.1132 \times 10^{-9} i$ & $0.19144+5.5701 \times 10^{-7} i$ & $0.17937+1.5825 \times 10^{-6} i$ \\
4 & $0.19910+4.2111 \times 10^{-9} i$ & $0.19400+4.4175 \times 10^{-7} i$ & $0.18398+1.7047 \times 10^{-6} i$ \\
5 & $0.19937+2.6307 \times 10^{-9} i$ & $0.19565+3.2839 \times 10^{-7} i$ & $0.18748+1.6231 \times 10^{-6} i$ \\
6 & $0.19954+1.7313 \times 10^{-9} i$ & $0.19674+2.3987 \times 10^{-7} i$ & $0.19012+1.4352 \times 10^{-6} i$ \\
7 & $0.19964+1.1917 \times 10^{-9} i$ & $0.19749+1.7591 \times 10^{-7} i$ & $0.19211+1.2145 \times 10^{-6} i$ \\
8 & $0.19972+8.5168 \times 10^{-10} i$ & $0.19801+1.3074 \times 10^{-7} i$ & $0.19362+1.0027 \times 10^{-6} i$ \\
9 & $0.19977+6.2818 \times 10^{-10} i$ & $0.19839+9.8786 \times 10^{-8} i$ & $0.19577+8.1840 \times 10^{-7} i$ \\
10 & $0.19981+4.7578 \times 10^{-10} i$ & $0.19868+7.5958 \times 10^{-8} i$ & $0.19566+6.6543 \times 10^{-7} i$ \\
\hline \hline
\end{tabular}

TABLE II. The explicit complex frequencies of low-lying overtone states with $m_{p}=\frac{2}{10}, q_{1}=\frac{2}{10}, q_{2}=0$, and $Q_{1}=1$ in the $\left(N_{1}, N_{2}\right)=(1,3)$ case.

\begin{tabular}{lccc}
\hline \hline$n$ & $\epsilon=2$ & $\epsilon=2.5$ & $\epsilon=3.5$ \\
\hline 1 & $0.17028+5.3213 \times 10^{-7} i$ & $0.16253+5.9330 \times 10^{-7} i$ & $0.15031+4.4746 \times 10^{-7} i$ \\
2 & $0.17769+1.0241 \times 10^{-6} i$ & $0.17036+1.2805 \times 10^{-6} i$ & $0.15810+1.1444 \times 10^{-6} i$ \\
3 & $0.18320+1.2619 \times 10^{-6} i$ & $0.17650+1.7653 \times 10^{-6} i$ & $0.16460+1.8423 \times 10^{-6} i$ \\
4 & $0.18728+1.2735 \times 10^{-6} i$ & $0.18131+1.9846 \times 10^{-6} i$ & $0.17005+2.3954 \times 10^{-6} i$ \\
5 & $0.19028+1.1522 \times 10^{-6} i$ & $0.18508+1.9873 \times 10^{-6} i$ & $0.17462+2.7524 \times 10^{-6} i$ \\
6 & $0.19250+9.7835 \times 10^{-7} i$ & $0.18804+1.8513 \times 10^{-6} i$ & $0.17846+2.9196 \times 10^{-6} i$ \\
7 & $0.19415+8.0070 \times 10^{-7} i$ & $0.19034+1.6454 \times 10^{-6} i$ & $0.18168+2.9307 \times 10^{-6} i$ \\
8 & $0.19537+6.4235 \times 10^{-7} i$ & $0.19215+1.4182 \times 10^{-6} i$ & $0.18439+2.8276 \times 10^{-6} i$ \\
9 & $0.19629+5.1069 \times 10^{-7} i$ & $0.19357+1.1985 \times 10^{-6} i$ & $0.18666+2.6498 \times 10^{-6} i$ \\
10 & $0.19699+4.0521 \times 10^{-7} i$ & $0.19469+1.0009 \times 10^{-6} i$ & $0.18856+2.4297 \times 10^{-6} i$ \\
\hline \hline
\end{tabular}


TABLE III. The explicit coefficients of the fitting functions in Eq. (4.12).

\begin{tabular}{lllllllll}
\hline \hline$\left(N_{1}, N_{2}\right)$ & $\epsilon$ & $\omega_{r}^{0}$ & $a_{1}$ & $b_{1}$ & $\omega_{i}^{0}$ & $a_{2}$ & $b_{2}$ & $c_{2}$ \\
\hline$(2,2)$ & 2.5 & 0.0275 & 4.87 & 4.26 & $3.31 \times 10^{-9}$ & 0.0328 & 0.174 & 0.0153 \\
& 5 & 0.0165 & 0.977 & 0.783 & $3.00 \times 10^{-8}$ & 0.484 & 0.0577 & 0.0157 \\
& 8 & 0.0107 & 0.417 & 0.299 & $2.17 \times 10^{-8}$ & 0.984 & 0.0667 & 0.00352 \\
$(1,3)$ & 2 & 0.0156 & 0.476 & 0.356 & $3.23 \times 10^{-8}$ & 0.814 & 0.0753 & 0.00510 \\
& 2.5 & 0.0130 & 0.348 & 0.243 & $2.81 \times 10^{-8}$ & 1.03 & 0.0756 & 0.00236 \\
& 3.5 & 0.00963 & 0.230 & 0.144 & $1.41 \times 10^{-8}$ & 1.40 & 0.0530 & 0.000548 \\
\hline \hline
\end{tabular}

quasibound states, $\omega_{r}$ is also a monotonously increasing function of $n$ with the ground state $(n=1)$ having the lowest frequency. On the other hand, $\omega_{i}$ does not change monotonously as we increase $n$, indicating that the most unstable mode in general is neither the ground state, nor the most excited state. Intriguingly, we find that the $n$ dependence of complex frequency can be accurately fitted with some simple close-form functions:

$$
\begin{aligned}
& \frac{\omega_{r}}{M}=\frac{1+a_{1} n}{1+b_{1} n} \omega_{r}^{0}, \\
& \frac{\omega_{i}}{M}=\frac{1+a_{2} n^{2}}{1+b_{2} n^{2}+c_{2} n^{4}} \omega_{i}^{0}, \quad(n=1,2,3, \ldots) .
\end{aligned}
$$

The coefficients $\left(\omega_{r_{0}}, a_{1}, b_{1}, \omega_{i_{0}}, a_{2}, b_{2}\right)$ depend on all the variables of the theory and the charge parameters of the solution. For the dataset in Tables I and II, we give these coefficients in Table III.

In Appendix C, we present Figs. 4 and 5, where we plot the dimensionless real and imaginary frequencies $\omega_{r} / M$ and $\omega_{i} / M$ with respect to the overtone number $n$ for various $\epsilon$ 's. The dots are the actual numerical results for the low-lying overtone numbers and the solid lines are produced by the fitting functions. As we can see from Fig. 4 and Fig. 5, our conjectured formulas in (4.12) match with the quantized frequency strikingly well. The results show that $\omega_{r}(n)$ increases monotonously while $\omega_{i}(n)$ can have a maximum. The results also indicate that for large $n$, we have

$$
\omega_{r}-\omega_{r}^{0} \sim-\frac{1}{n}, \quad \omega_{i} \sim \frac{\omega_{i}^{0}}{n^{2}} .
$$

Thus, the instability is not caused by modes with large overtones.

We now turn to the discussion of how $\omega_{i}$ depends on the charge configuration, described by the dimensionless parameter $\epsilon$. This is important since we have already seen that there is no quasibound state when $\epsilon=0$. We thus naturally expect that quasibound states would emerge only for sufficiently large $\epsilon$. We focus our discussion for overtone numbers $n=1$ and $n=2$. We also fixed parameters $m_{p}=\frac{2}{10}$ and $q_{1}=\frac{2}{10}$ and $q_{2}=0$. We present our results in Fig. 6 of Appendix C. To better describe the $\epsilon$ dependence, we find that the numerical data can be fitted by an exponential function, given by

$\log \frac{\omega_{i}}{M}=a_{3} \epsilon^{-\frac{N_{1}+N_{2}}{2}}+b_{3} \epsilon^{-\frac{N_{2}}{2}}+c_{3} \epsilon^{-\frac{N_{1}}{2}}+d_{3}+e_{3} \epsilon^{\frac{N_{1}}{2}}+f_{3} \epsilon^{\frac{N_{2}}{2}}$.

In the case of $\left(N_{1}, N_{3}\right)=(1,3)$, the values of the coefficients $\left(a_{3}, b_{3}, c_{3}, d_{3}, e_{3}, f_{3}\right)$ are given in Table IV.

For the case of $\left(N_{1}, N_{2}\right)=(2,2)$, Eq. (4.14) reduces to a simpler function:

$$
\log \frac{\omega_{i}}{M}=\tilde{a}_{3} \epsilon^{-2}+\tilde{b}_{3} \epsilon^{-1}+\tilde{c}_{3}+\tilde{d}_{3} \epsilon .
$$

The coefficients $\left(\tilde{a}_{3}, \tilde{b}_{3}, \tilde{c}_{3}, \tilde{d}_{3}\right)$ are given in Table V.

Our numerical data of $\omega_{i}$ run from $10^{-12}$ to $10^{-6}$ as $\epsilon$ increases from a small value to a larger one, and that is quite a few orders of magnitude. For this reason, we plot our results in Fig. 6, using logarithmic $\log \left(\omega_{i} / M\right)$. The plots illustrate that our conjectured curve functions fit with the data remarkably well.

We now examine the example of $\left(N_{1}, N_{2}\right)=(2,2)$ and $n=1$ in further detail. We have computed quasibound states for parameter $\epsilon$ from about 1 to 12 . We see that the imaginary part of the dimensionless frequency has a maximum of $\sim 10^{-6}$ around $\epsilon \sim 6$, and it becomes vanishingly small $\left(\epsilon \sim 10^{-12}\right)$ as $\epsilon \sim 1$. This appears to have confirmed our earlier expectation that quasibound states

TABLE IV. The value of $\left(a_{3}, b_{3}, c_{3}, d_{3}, e_{3}, f_{3}\right)$ for the fitting function Eq. (4.14) in the $\left(N_{1}, N_{2}\right)=(1,3)$ case.

\begin{tabular}{ccccccc}
\hline \hline$n$ & $a_{3}$ & $b_{3}$ & $c_{3}$ & $d_{3}$ & $e_{3}$ & $f_{3}$ \\
\hline 1 & 8.43 & -21.5 & 9.62 & -9.65 & -6.49 & 0.295 \\
2 & 21.5 & -59.0 & 93.5 & -99.3 & 25.1 & -1.04 \\
\hline \hline
\end{tabular}

TABLE V. The value of coefficients $\left(\tilde{a}_{3}, \tilde{b}_{3}, \tilde{c}_{3}, \tilde{d}_{3}\right)$ for the fitting function Eq. (4.15) in the $\left(N_{1}, N_{2}\right)=(2,2)$ case.

\begin{tabular}{ccccc}
\hline \hline$n$ & $\tilde{a}_{3}$ & $\tilde{b}_{3}$ & $\tilde{c}_{3}$ & $\tilde{d}_{3}$ \\
\hline 1 & -1.97 & -16.9 & -11.5 & 0.422 \\
2 & 2.09 & -22.9 & -10.2 & -0.403 \\
\hline \hline
\end{tabular}


cease to exist for sufficiently small $\epsilon$. This feature remains true for the $n=2$ states, or for the $\left(N_{1}, N_{2}\right)=(1,3)$ theory.

However, there is a caveat in our above discussion, since we set $q_{2}=0$. We might naively conclude that the black holes with small enough $\epsilon$ might be exempted from superradiant instability. This obviously appears to be consistent with the fact that the RN black hole $(\epsilon=0)$ is stable. However, the situation changes if we turn on the $q_{2}$ parameter. To illustrate this, we consider the $\left(N_{1}, N_{2}\right)=$ $(2,2)$ example and the leading falloff of the large- $r$ expansion of the effective potential is

$$
V_{\mathrm{eff}}=m_{p}^{2}+\frac{\beta}{r}+\mathcal{O}\left(1 / r^{2}\right),
$$

where

$$
\begin{aligned}
& \beta=2 Q_{1}\left(2 \sqrt{2}(x+y \omega)+\epsilon\left(\sqrt{2}\left(x-\omega^{2}\right)+4 q_{2} \omega\right)\right), \\
& x=m_{p}^{2}-\omega^{2}>0, \quad y=\sqrt{2}\left(q_{1}+q_{2}\right)-\omega>0 .
\end{aligned}
$$

As was explained earlier, for $V_{\text {eff }}$ to have one peak, we must have $\beta<0$. This is not possible for the RN black hole with $\epsilon=0$. When $q_{2}=0$, we find that $\beta$ can only be negative for sufficiently large $\epsilon$ since $\omega<m_{p}$. However, if we turn on $q_{2}$ and let it be negative, then we can have negative $\beta$ even for smaller $\epsilon$. Of course, the condition $y>0$ restricts how negative $q_{2}$ can go. Analogous results can be found for the $\left(N_{1}, N_{2}\right)=(1,3)$ theory.

It becomes clear why trapping wells can exist in STU black holes while they do not in the RN black hole. Having negative $\beta$ is a sign that the overall force becomes attractive at the asymptotic infinity, and this requirement can be arranged in the STU theory since we can have additional antiparticles with negative $q_{2}$ that is attractive when it interacts with $Q_{2}$. These attractive components, mediated also through the dilaton scalar, create an effective potential with a trapping well. This also implies that superradiant instability could exist as long as the STU black hole has not degenerated to become the RN black hole and hence the dilatonic scalars are not vanishing.

However, having found the condition for negative $\beta$ provides only the necessary condition for the quasibound states and we still resort to numerical calculations for more quasibound states. We start from the least unstable state among those in Fig. 6, namely $\epsilon=1$ and $q_{2}=0$, for which we have seen that $\omega_{i} / M \sim 10^{-14}$ is vanishingly small. Figure 6 appears to suggest that black holes with smaller $\epsilon$, e.g., $\epsilon=0.5$, would become stable. However, if we fix the parameters $m_{p}=\frac{2}{10}, q_{1}=\frac{2}{10}, Q_{1}=1$, and $\epsilon=1$, but turn on $q_{2}$ so that $q_{2} / q_{1}$ runs from 0 to some small negative number, we find that $\omega_{i} / M$ dramatically increases by a few orders of magnitude. Explicitly, from the panels of the first row in Fig. 7 of Appendix C, in which we plot the $\omega_{i} / M$ in terms of the fundamental charge ratio of $q_{2} / q_{1}$. We see that
TABLE VI. The value of coefficients $\left(\tilde{a}_{3}, \tilde{b}_{3}, \tilde{c}_{3}, \tilde{d}_{3}\right)$ for the fitting function Eq. (4.15) in $\left(N_{1}, N_{2}\right)=(2,2), q_{2}=-0.048$ case.

\begin{tabular}{ccccc}
\hline \hline$n$ & $\tilde{a}_{3}$ & $\tilde{b}_{3}$ & $\tilde{c}_{3}$ & $\tilde{d}_{3}$ \\
\hline 1 & 0.247 & -3.35 & -24.1 & 5.53 \\
2 & 0.1819 & -2.92 & -26.1 & 6.33 \\
\hline \hline
\end{tabular}

when this ratio is zero, $\omega_{i} / M$ is vanishingly small, around $10^{-14}$. However, it increases from $10^{-14}$ to around the maximum $10^{-10}$ and then decreases again, as we make the ratio more and more negative.

We therefore select $q_{2}=-0.048$, corresponding to the maximum of $\omega_{i} / M \approx 3.8 \times 10^{-10}$, and fix also parameters $m_{p}=\frac{2}{10}, q_{1}=\frac{2}{10}, Q_{1}=1$. We construct more quasibound states by decreasing $\epsilon$ from 1 to some smaller number. We find that the instability modes exist for smaller $\epsilon$, with $\omega_{i} / M$ decreasing to $10^{-13}$ as $\epsilon$ runs from 1 to around 0.3 . Similarly, in the case of $q_{2}=-0.048$, the fitting functions in Eq. (4.15) for $\omega_{i} / M$ with respect to $\epsilon$ are also valid, and the panels of the second row of Fig. 7 show that they fit remarkably well. The coefficients $\left(\tilde{a}_{3}, \tilde{b}_{3}, \tilde{c}_{3}, \tilde{d}_{3}\right)$ are explicitly given in Table VI.

The above results strongly suggest that STU black holes with nonvanishing $\epsilon$ are always unstable. Indeed, from (4.17), we can see that no matter how small $\epsilon \neq 0$ is, we can always increase $q_{1}$ positively and make $q_{2}$ more negative so that $\beta$ becomes negative while keeping $q_{1}+q_{2}$ fixed to satisfy the superradiant condition. Concretely, we obtain unstable quasibound states for $Q_{1}=1, \epsilon=0.01$, with the complex frequency

$$
\omega=0.1927+3.752 \times 10^{-8} i,
$$

when we choose $m_{p}=\frac{2}{10}, q_{1}=20.2, q_{2}=-20$. We may therefore conclude that the superradiant stability of the RN black hole is a fine-tuning result from the perspective of the STU supergravity model.

\section{Effective potential with double peaks}

As was discussed earlier, at large $r$, the leading falloff of $V_{\text {eff }}$ takes the form of (4.16). In the previous subsection, we consider the case with $\beta<0$, in which case the potential has a single peak. Here, we consider the possibility of $\beta>0$, in which case a potential trapping well necessarily involves two peaks. For simplicity, we only investigate the $\left(N_{1}, N_{2}\right)=(2,2)$ example. A simple way to achieve $\beta>0$ is to take $q_{2}=q_{1}$, in which case we have

$$
\beta=2 \sqrt{2}\left(Q_{1}+Q_{2}\right) \omega^{2}\left(x^{2}+2 x+y\right),
$$

where $x>0$ and $y>0$ are defined by (3.24) with $\omega_{c}=2 \sqrt{2} q_{1}$. As we have shown, for the RN black hole 
TABLE VII. The explicit $\left(\omega_{r_{0}}, a_{1}, b_{1}, \omega_{i_{0}}, a_{2}, b_{2}\right)$ for Eq. (4.12) in the $\left(N_{1}, N_{2}\right)=(2,2)$ case, with $m_{p}=\frac{5}{100}, q_{1}=q_{2}=\frac{2}{100}$, $Q_{2}=100$. The corresponding $\omega_{r}(n)$ and $\omega_{i}(n)$ produce the solid lines in Fig. 8.

\begin{tabular}{llllllll}
\hline \hline$\epsilon$ & $\omega_{r}^{0}$ & $a_{1}$ & $b_{1}$ & $\omega_{i}^{0}$ & $a_{2}$ & $b_{2}$ & $c_{2}$ \\
\hline 99 & $2.90 \times 10^{-4}$ & 0.147 & 0.101 & $2.49 \times 10^{-9}$ & 0.76 & 0.0271 \\
999 & $2.58 \times 10^{-4}$ & 0.0794 & 0.0344 & $1.59 \times 10^{-8}$ & 0.621 & 0.000807 \\
\hline \hline
\end{tabular}

TABLE VIII. The value of coefficients $\left(\tilde{a}_{3}, \tilde{b}_{3}, \tilde{c}_{3}, \tilde{d}_{3}\right)$ for the fitting function Eq. (4.15) in the $\left(N_{1}, N_{2}\right)=(2,2)$ case, with $m_{p}=\frac{5}{100}, q_{1}=q_{2}=\frac{2}{100}, Q_{2}=100$.

\begin{tabular}{lrrrr}
\hline \hline$n$ & $\tilde{a}_{3}$ & $\tilde{b}_{3}$ & $\tilde{c}_{3}$ & $\tilde{d}_{3}$ \\
\hline 1 & -197 & -184 & -17.3 & -0.000116 \\
2 & 132 & -203 & -16.5 & -0.000163 \\
\hline \hline
\end{tabular}

with $Q_{2}=Q_{1}$, the potential $V_{\text {eff }}$ shapes like Fig. 2 and therefore has no trapping well. It turns out that if we take an alternative limit with $Q_{1}=0$ (or equivalently $Q_{2}=0$ ), there is no trapping well either. However, trapping well does arise when we keep $Q_{2} / Q_{1}$ sufficiently large, but not too large, corresponding to a large but not too large $\epsilon$, defined by (4.9).

In what follows, we illustrate the superradiant instability associated with quasibound states in the trapping-well potential with double peaks. We fix parameters $m_{p}=\frac{5}{100}, q_{1}=q_{2}=\frac{2}{100}, Q_{2}=100$, and let $Q_{1}$ run from 1 to 0.04 , corresponding to $\epsilon$ running from 99 to 2499.

We begin with presenting the quasibound states with fixed $Q_{1}=1(\epsilon=99)$ and $Q_{1}=0.1(\epsilon=999)$ for some low-lying overtone number $n$. We find that the complex frequency as a function of $n$ can also be fitted with our conjectured formulas in Eq. (4.12), with coefficients $\left(\omega_{r_{0}}, a_{1}, b_{1}, \omega_{i_{0}}, a_{2}, b_{2}\right)$ given in Table VII. As we can see from Fig. 8, the solid lines drawn by the fitting functions match with the numerical data quite well.

Finally, we turn to investigate how the dimensionless instability parameter $\omega_{i} / M$ changes according to the value of $\epsilon$. Similarly, we study only the ground state $(n=1)$ and the first-excited state $(n=2)$ as examples. As was mentioned, we expect that the quasibound states do not exist for small $\epsilon$ and neither for $\epsilon$ that is too large. In Fig. 9, we present $\log \left(\omega_{i} / M\right)$ as functions of $\epsilon$ that runs from 99 to 2499. Even though we have obtained a large region of $\epsilon$, we find that our conjectured formula Eq. (4.15) with coefficients given in Table VIII can fit the data perfectly well.

\section{CONCLUSIONS}

In this paper, we studied superradiant instability by constructing quasibound states of charged Klein-Gordon scalar equation in the background of extremal charged black holes of the STU supergravity model. The general black hole carries four $U(1)$ charges and when they are all equal, it reduces to the RN black hole. We were motivated by the fact that the RN black hole seems to be exempted from the superradiant instability and we would like to study this phenomenon from the perspective of these more general charged black holes.

We first addressed some theoretical aspects of the subject. We proved that all quasibound states, as well as the quasinormal modes, necessarily involve complex frequencies. This motivated us to establish superradiant boundary conditions for such states with complex frequencies. Owing to the extra $U(1)$ fields and scalars in the STU model, the effective potential of the Schrödinger-like equation governing the radial wave function has far richer structure than the one associated with the RN black hole. For simplicity, we reduced the general STU model to involve only one scalar and two $U(1)$ fields. The reduced system with the nontrivial dilaton captures the essence of the STU model.

We found that for suitable parameters, there exist two types of trapping wells: those with single peak and those with double peaks. Using the numerical shooting method, we found that both potential wells could trap quasibound states with positive imaginary frequency, indicating superradiant instability. These are the first examples in literature where superradiant instability is demonstrated for spherically symmetric and static black holes. We obtained how the complex frequency of the quasibound states depended on the overtone number, as well as the parameter $\epsilon$ that measures the deviation of the STU black holes from the RN black hole.

Our results indicate that STU charged black holes are superradiant unstable as long as the charges are not equal. In other words, no matter how small $\epsilon$ is, we can always find superradiant instability associated with appropriate fundamental charged massive scalar particles and antiparticles. Therefore, the superradiant stability of the RN black hole is a fine-tuning result in the framework of the STU supergravity theory.

Our results appears to contradict the common belief that extremal charged black holes in STU supergravity, which preserve a certain fractions of supersymmetry, should be stable against perturbation. While it is certainly true that the charged massive scalar field $\Phi$ could arise in the KaluzaKlein spectrum when we examine the origin of the STU model from $M$ theory or strings, we expect that such a $\Phi$ should have nonminimal couplings with other massless scalar fields in the STU model. The toy model (2.7) is thus 
likely to break the supersymmetry. Nevertheless, the ease of having superradiant instability indicates that one should reexamine the issue further within supergravity. It is clear that the electric repulsion and gravity attraction associated with RN black hole and the superradiant particle is too simple for the effective potential to have a potential well. The scalar fields in the STU model can yield both attractive and repulsive long-ranged forces, and this makes it possible for creating a potential trapping well. It is thus of great interest to study generally how scalar fields affect the superradiant (in)stability of black holes with the scalar hair.

\section{ACKNOWLEDGMENTS}

We thank Bing Sun for useful discussions. This work is supported by the National Natural Science Foundation of China (NSFC) Grants No. 12005155, No. 11875200, and No. 11935009, and also No. 11947301 and No. 12047502.

\section{APPENDIX A: TORTOISE COORDINATES}

To investigate the asymptotic behavior of quasibound states, it is useful to introduce the following definition of tortoise coordinate $y$ and radial function transformation $\bar{R}$ :

$\frac{\mathrm{d} y}{\mathrm{~d} r}=\left(\tilde{H}_{1} \tilde{H}_{2} \tilde{H}_{3} \tilde{H}_{4}\right)^{\frac{1}{2}}, \quad \bar{R} \equiv\left(\tilde{H}_{1} \tilde{H}_{2} \tilde{H}_{3} \tilde{H}_{4}\right)^{-1 / 4} \frac{R(r)}{r}$,

where $y \rightarrow-\infty$ as $r \rightarrow 0$ while $y \rightarrow \infty$ as $r \rightarrow \infty$. Then the radial equation can be written in standard wave equation

$-\frac{\mathrm{d}^{2} \bar{R}}{\mathrm{~d} y^{2}}+\bar{U}(y) \bar{R}=0, \quad \bar{U}=-(\omega-Q(y))^{2}+V(y)$,

where

$$
\begin{aligned}
& Q(y)=\sum_{i=1}^{4} q_{i}\left(1-\tilde{H}_{i}^{-1}\right), \\
& V(y)=\frac{\ell(\ell+1)}{r^{2} \tilde{H}}+\frac{m_{p}^{2}}{\sqrt{\tilde{H}}}-\frac{5 \tilde{H}^{\prime}}{16 \tilde{H}^{3}}+\frac{\tilde{H}^{\prime \prime}}{4 \tilde{H}^{2}}, \quad \tilde{H}=\prod_{i=1}^{4} \tilde{H}_{i} .
\end{aligned}
$$

The potential $\bar{U}$ has the following asymptotic behavior:

$$
\left.\bar{U}\right|_{y \rightarrow-\infty} \sim-\left(\omega-\omega_{c}\right)^{2}, \quad \bar{U}_{y \rightarrow \infty}=m_{p}^{2}-\omega^{2}>0 .
$$

With such asymptotic behavior of $\tilde{U}$, we will arrive at the asymptotic solution of $\tilde{R}$ that

$$
\bar{R} \propto \begin{cases}\mathrm{e}^{-i\left(\omega-\omega_{c}\right) y} & y \rightarrow-\infty \\ \mathrm{e}^{-\sqrt{m_{p}^{2}-\omega^{2}} y} & y \rightarrow \infty,\end{cases}
$$

where we discarded the divergent mode and the wave function decays exponentially at infinity, giving rise to a quasibound state. Compared to the original $r$ variable, in the two asymptotic regions, we have

$y \sim-\frac{1}{r}, \quad \bar{R} \sim R, \quad$ as $r \rightarrow 0 ; \quad y \sim r$,

$\bar{R} \sim \frac{1}{r} R, \quad$ as $r \rightarrow \infty$.

Equation (A5) can then be translated to the boundary conditions in $r$ coordinate, namely, Eq. (2.11). Thus, we see that $r=0$ horizon with ingoing boundary condition is not an infinite potential barrier, but a nonrepulsive asymptotic region.

It is worth remarking that when $\omega>m_{p}$, the function $\bar{R}$ becomes wavelike also as $y \rightarrow \infty$. A quasinormal mode is defined as the one with outgoing boundary condition only, namely, $\bar{R} \sim \mathrm{e}^{i \sqrt{\omega^{2}-m_{p}^{2}} y}$ as $y \rightarrow \infty$.

\section{APPENDIX B: A PROOF OF COMPLEX FREQUENCIES}

Theorem 1. The time frequency $\omega$ of quasinormal modes or quasibound states cannot be a real number.

Proof.-We begin by assuming that the frequency of quasinormal modes or quasibound states is a real number. By performing integration by parts on the radial equation Eq. (A2), we have

$\left.\bar{R}_{n}^{*} \frac{\mathrm{d} \bar{R}_{n}}{\mathrm{~d} y}\right|_{-\epsilon} ^{\epsilon}=\int_{-\epsilon}^{\epsilon}\left(\left|\bar{R}_{n}^{\prime}\right|^{2}-\left(\left(\omega_{n}-Q(y)\right)^{2}-V(y)\right)\left|\bar{R}_{n}\right|^{2}\right) \mathrm{d} y$,

where a prime denotes a derivative with respect to $y$. We set $\epsilon \gg 1$, but not infinity, to avoid divergence. With boundary conditions that define either the quasinormal modes or quasibound states, the left-hand side of Eq. (B1) gives

quasinormal modes: $\left.\bar{R}_{n}^{*} \frac{\mathrm{d} \bar{R}_{n}}{\mathrm{~d} y}\right|_{-\epsilon} ^{\epsilon}$

$$
=i\left(\left|c_{1}\right|^{2}\left(\omega-\omega_{c}\right)+\left|c_{2}\right|^{2} \sqrt{\omega^{2}-m_{p}^{2}}\right), \quad m_{p}^{2}<\omega^{2}
$$

quasibound states: $\left.\bar{R}_{n}^{*} \frac{\mathrm{d} \bar{R}_{n}}{\mathrm{~d} y}\right|_{-\epsilon} ^{\epsilon}$

$$
=i\left|c_{1}\right|^{2}\left(\omega-\omega_{c}\right), \quad m_{p}^{2}>\omega^{2},
$$

which is purely imaginary. On the other hand, the righthand side of Eq. (B1) must be real, since $\omega, Q(y)$ and $V(y)$ are all real, i.e.,

$\int_{-\epsilon}^{\epsilon}\left(\left|\bar{R}_{n}^{\prime}\right|^{2}-\left(\left(\omega_{n}-Q(y)\right)^{2}-V(y)\right)\left|\bar{R}_{n}\right|^{2}\right) \mathrm{d} y \in \mathbb{R}$.

The contradiction shows that neither the quasinormal modes nor quasibound states can have a real time 

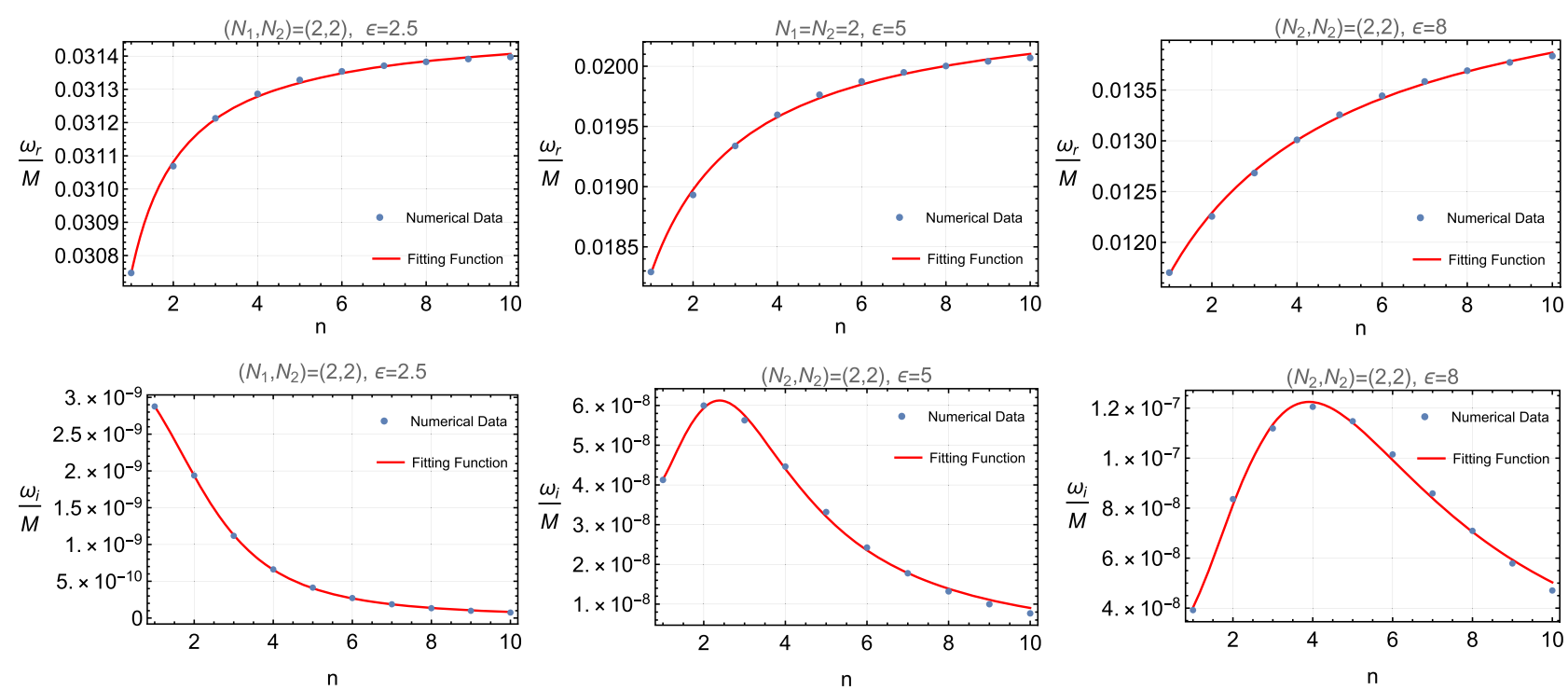

FIG. 4. Here we plot the $\omega_{r} / M$ and $\omega_{i} / M$ with respect to the overtone $n$ for various $\epsilon$, for the case of $\left(N_{1}, N_{2}\right)=(2,2)$. The solid lines are produced by the corresponding fitting functions in (4.12). We see that our fitting functions match the data remarkably well. Fixed parameters are $m_{p}=\frac{2}{10}, q_{1}=\frac{2}{10}, q_{2}=0$, and $Q_{1}=1$.

frequency. It should be pointed that our proof relies only on the boundary conditions of either quasinormal modes or quasibound states. It does not depend on the details such as $V(y)$ and $Q(y)$, and therefore the statement is completely general.

\section{APPENDIX C: PLOTS AND FIGURES}

In this appendix, we give our plots and figures discussed in Sec. IV. The explanations of graphs are provided by the captions and also the associated discussions in the main text.
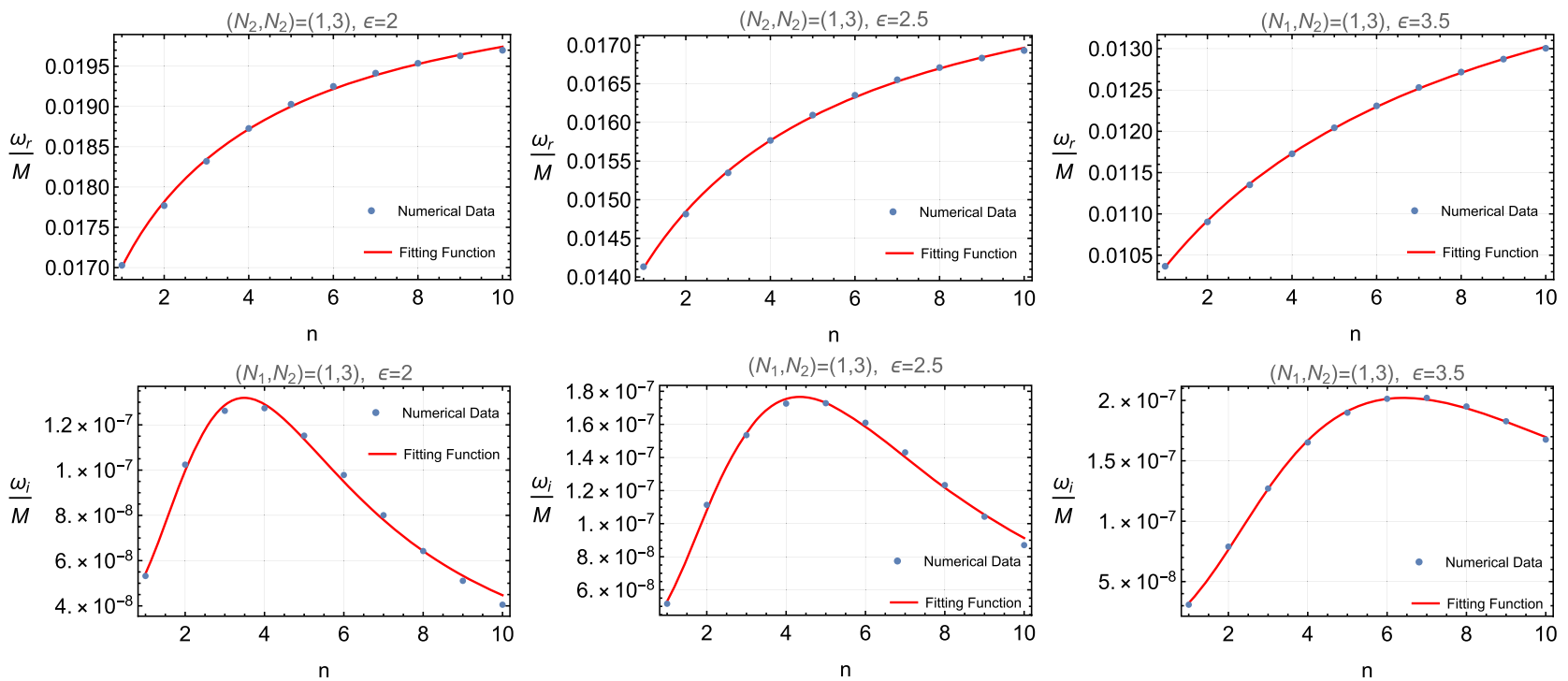

FIG. 5. Here we plot the $\omega_{r} / M$ and $\omega_{i} / M$ with respect to the overtone $n$ for various $\epsilon$, for the case of $\left(N_{1}, N_{2}\right)=(1,3)$. The solid lines are produced by the corresponding fitting functions in (4.12). We see that our fitting functions match the data remarkably well. Fixed parameters are $m_{p}=\frac{2}{10}, q_{1}=\frac{2}{10}, q_{2}=0$, and $Q_{1}=1$. 


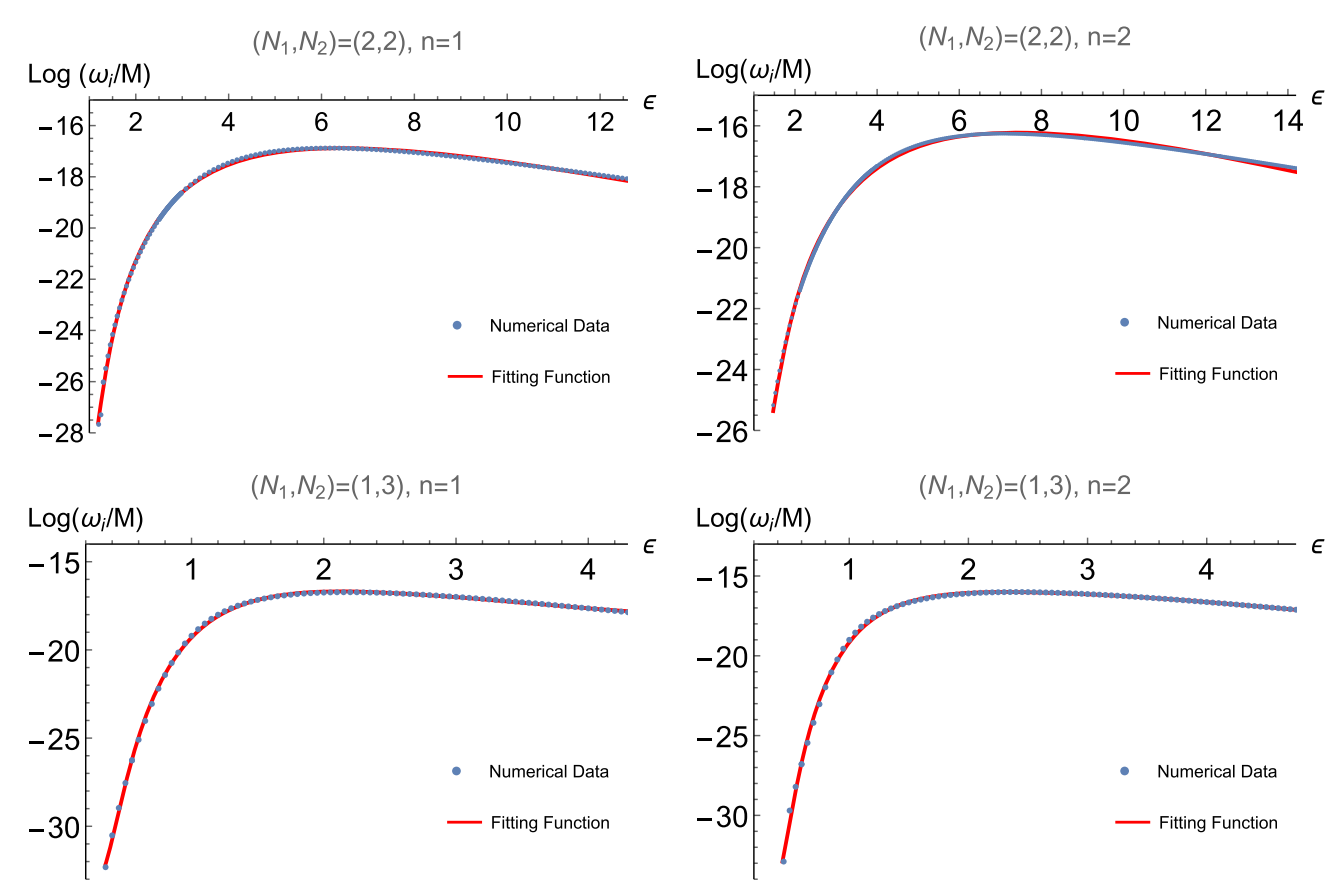

FIG. 6. The plot of $\log \left(\omega_{i} / M\right)$ with respect to $\epsilon$ in $\left(N_{1}, N_{2}\right)=(2,2)$ and $\left(N_{1}, N_{2}\right)=(1,3)$ examples. Fixed parameters are $m_{p}=\frac{2}{10}$, $q_{1}=\frac{2}{10}, q_{2}=0$, and $Q_{1}=1$.
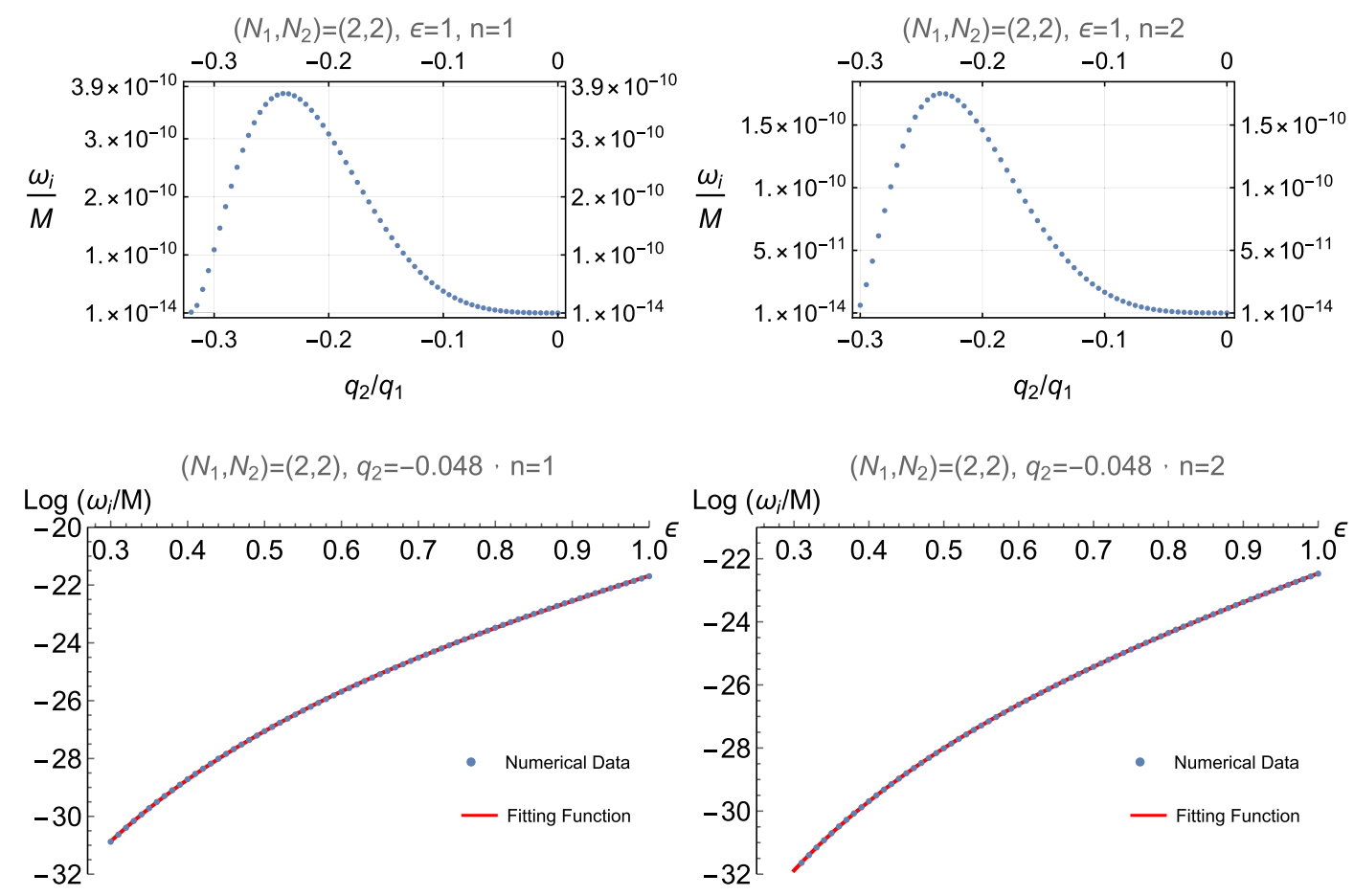

FIG. 7. In the first row, we plot the $\omega_{i} / M$ in terms of the fundamental charge ratio $q_{2} / q_{1}$, with fixed parameters $m_{p}=\frac{2}{10}, q_{1}=\frac{2}{10}$, $Q_{1}=1$, and $\epsilon=1$. We see that $\epsilon=1$ state can actually become quite unstable as we run $q_{2}$ towards some negative value, with $q_{2}=-0.048$ giving the maximum instability of order $10^{-10}$. In the second row, therefore choosing $q_{2}=-0.048$, we plot the $\log \omega_{i} / M$ with respect to $\epsilon$. This establishes that the $\epsilon=0.3$ black hole is also unstable. 

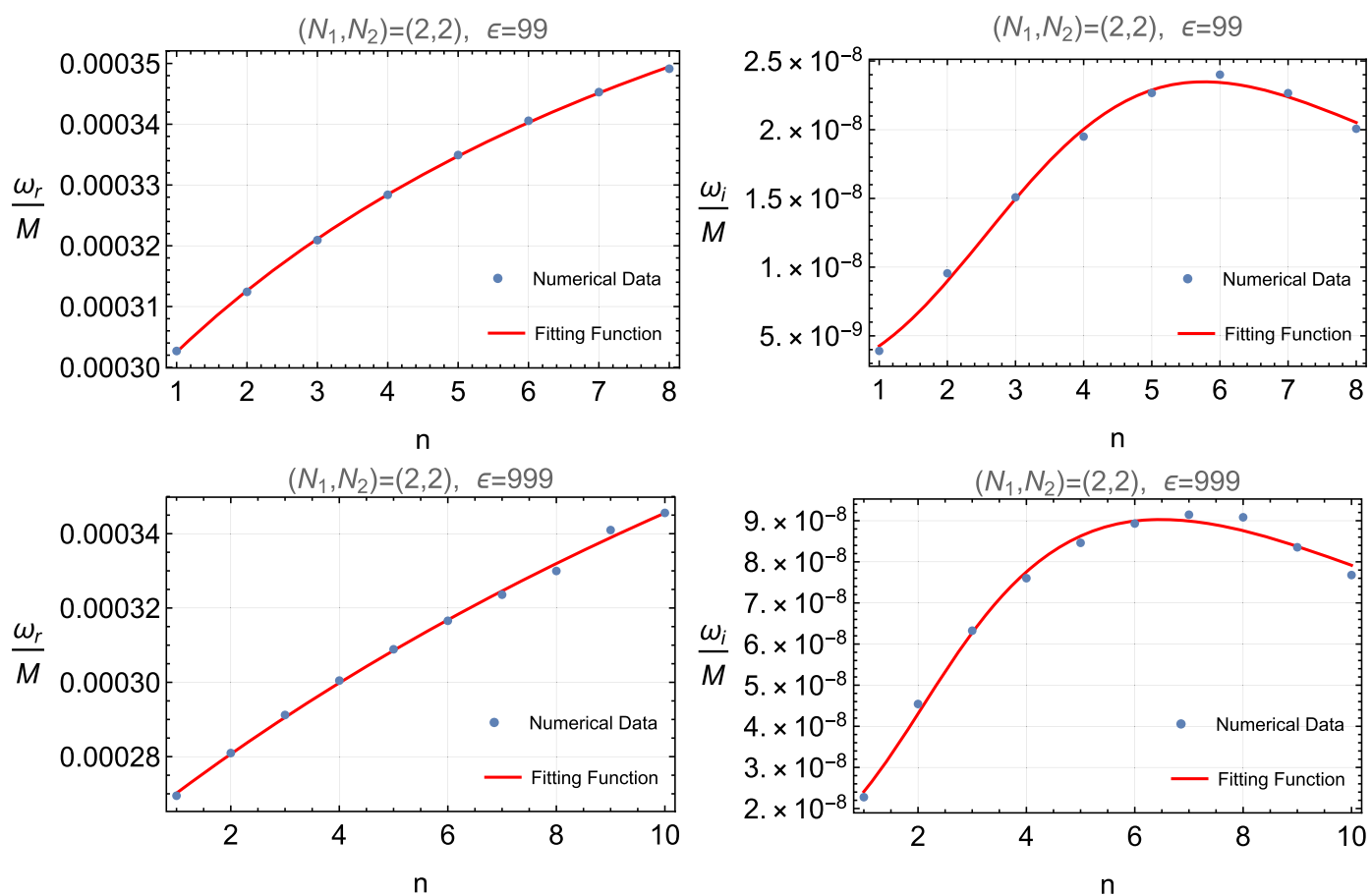

FIG. 8. For $\left(N_{1}, N_{2}\right)=(2,2)$, we give the $\omega_{r} / M$ and $\omega_{i} / M$ with respect to $n$ for $Q_{2}=1(\epsilon=99)$ and $0.1(\epsilon=999)$, with fixed $m_{p}=\frac{5}{100}, q_{1}=q_{2}=\frac{2}{100}, Q_{2}=100$. The solid lines are produced by the fitting functions in (4.12).

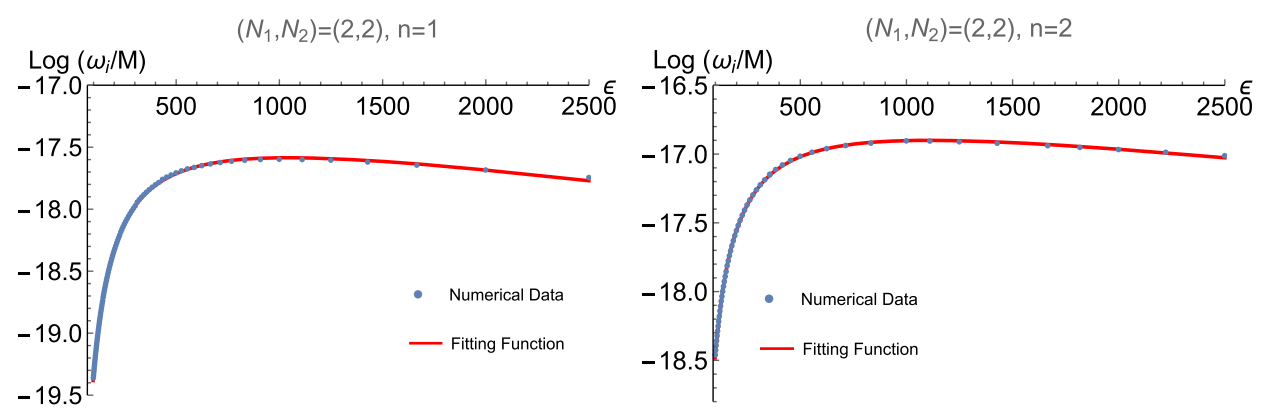

FIG. 9. For $\left(N_{1}, N_{2}\right)=(2,2)$, we plot $\log \left(\omega_{i} / M\right)$ as a function of $\epsilon$, with fixed $m_{p}=\frac{5}{100}, q_{1}=q_{2}=\frac{2}{100}, Q_{2}=100$. The dimensionless parameter $\epsilon$ runs from 99 to 2499 , corresponding to $Q_{1}$ running from 1 to 0.04 . The solid lines drawn from (4.15) match the numerical data remarkably well for such large expanse of $\epsilon$ value.

[1] B. P. Abbott et al. (LIGO Scientific, Virgo Collaborations), Observation of Gravitational Waves from a Binary Black Hole Merger, Phys. Rev. Lett. 116, 061102 (2016).

[2] K. Akiyama et al. (Event Horizon Telescope Collaboration), First M87 event horizon telescope results. I. The shadow of the supermassive black hole, Astrophys. J. Lett. 875, L1 (2019).
[3] W. H. Press and S. A. Teukolsky, Floating orbits, superradiant scattering and the black-hole Bomb, Nature (London) 238, 211 (1972).

[4] J. D. Bekenstein, Extraction of energy and charge from a black hole, Phys. Rev. D 7, 949 (1973).

[5] S. A. Teukolsky and W. H. Press, Perturbations of a rotating black hole. III-Interaction of the hole with gravitational and electromagnet ic radiation, Astrophys. J. 193, 443 (1974). 
[6] A. A. Starobinskil and S. M. Churilov, Amplification of electromagnetic and gravitational waves scattered by a rotating "black hole", Sov. Phys. JETP 65, 1 (1974).

[7] A. A. Starobinsky, Amplification of waves reflected from a rotating "black hole", Sov. Phys. JETP 37, 28 (1973).

[8] R. Brito, V. Cardoso, and P. Pani, Superradiance: New frontiers in black hole physics, Lect. Notes Phys. 906, 1 (2015).

[9] T. Damour, N. Deruelle, and R. Ruffini, On quantum resonances in stationary geometries, Lett. Nuovo Cimento 15, 257 (1976).

[10] S. L. Detweiler, Klein-Gordon equation and rotating black holes, Phys. Rev. D 22, 2323 (1980).

[11] H. Furuhashi and Y. Nambu, Instability of massive scalar fields in Kerr-Newman space-time, Prog. Theor. Phys. 112, 983 (2004).

[12] S. R. Dolan, Instability of the massive Klein-Gordon field on the Kerr spacetime, Phys. Rev. D 76, 084001 (2007).

[13] Y. Huang, D.-J. Liu, X.-h. Zhai, and X.-z. Li, Instability for massive scalar fields in Kerr-Newman spacetime, Phys. Rev. D 98, 025021 (2018).

[14] V. Cardoso and O. J. C. Dias, Small Kerr-anti-de Sitter black holes are unstable, Phys. Rev. D 70, 084011 (2004).

[15] V. Cardoso, O. J. C. Dias, J. P. S. Lemos, and S. Yoshida, The black hole bomb and superradiant instabilities, Phys. Rev. D 70, 044039 (2004).

[16] R. Li, Superradiant instability of charged massive scalar field in Kerr-Newman-anti-de Sitter black hole, Phys. Lett. B 714, 337 (2012).
[17] M. Wang and C. Herdeiro, Superradiant instabilities in a D-dimensional small Reissner-Nordström-anti-de Sitter black hole, Phys. Rev. D 89, 084062 (2014).

[18] P. Bosch, S. R. Green, and L. Lehner, Nonlinear Evolution and Final Fate of Charged Anti-de Sitter Black Hole Superradiant Instability, Phys. Rev. Lett. 116, 141102 (2016).

[19] B. Ganchev, Superradiant instability in AdS, arXiv:1608 .01798 .

[20] S. Hod, Stability of the extremal Reissner-Nordstroem black hole to charged scalar perturbations, Phys. Lett. B 713, 505 (2012).

[21] J.-H. Huang and Z.-F. Mai, Superradiantly stable nonextremal Reissner-Nordström black holes, Eur. Phys. J. C 76, 314 (2016).

[22] S. Hod, Stability of highly-charged Reissner-Nordström black holes to charged scalar perturbations, Phys. Rev. D 91, 044047 (2015).

[23] M. J. Duff, J. T. Liu, and J. Rahmfeld, Four-dimensional string-string-string triality, Nucl. Phys. B459, 125 (1996).

[24] M. Cvetič, M. J. Duff, P. Hoxha, J. T. Liu, H. Lü, J. X. Lu, R. Martinez-Acosta, C. N. Pope, H. Sati, and T. A. Tran, Embedding AdS black holes in ten-dimensions and eleven-dimensions, Nucl. Phys. B558, 96 (1999).

[25] H. Lü, Charged dilatonic ads black holes and magnetic $\mathrm{AdS}_{D-2} \times R^{2}$ vacua, J. High Energy Phys. 09 (2013) 112.

[26] L. Di Menza and J.-P. Nicolas, Superradiance on the Reissner-Nordstrøm metric, Classical Quantum Gravity 32, 145013 (2015). 